\title{
SDO AIA and Hinode EIS observations of "warm" loops
}

\author{
G. Del Zanna, B. O’Dwyer, and H. E. Mason
}

\author{
DAMTP, Centre for Mathematical Sciences, University of Cambridge, Wilberforce Road, Cambridge CB3 0WA UK \\ e-mail: g.del-zanna@damtp.cam.ac.uk
}

Received 13 June 2011 / Accepted 7 September 2011

\begin{abstract}
We present simultaneous observations of active region "warm" (1 MK) loops using the Solar Dynamics Observatory (SDO) Atmospheric Imaging Assembly (AIA) and Hinode EUV Imaging Spectrometer (EIS). Sample EIS spectra for a loop footpoint and a lop leg region are presented, and are used to describe the spectral lines which contribute to the six AIA EUV channels, both directly and predicted with DEM modeling. We find good overall agreement between observed and predicted count rates for the $131 \AA$, $193 \AA$, and $335 \AA$ bands, but highlight a number of problems, partly to be ascribed to inter-calibration issues, partly due to the fact that a large number of lines remain unidentified for the $94 \AA, 171 \AA$, and $211 \AA$ bands. We also found that the $335 \AA$ band is severely affected by cross-talk with the $131 \AA$ band and by second order contributions. We extend our previous work where we highlighted the multi-thermal nature of the SDO AIA bands to show that emission from lines formed at typical transition region temperatures $(\log T[\mathrm{~K}]=5.0-5.8)$ can be significant for all the EUV channels, and even dominant in some cases. We also assess the possibility of deriving accurate emission measures from the AIA observations. We have found that the inversion of the AIA data to obtain a description of the thermal characteristics of warm loops is unreliable. We highlight the need for further work on the relevant atomic data before the AIA data can be reliably used for plasma diagnostic purposes.
\end{abstract}

Key words. techniques: spectroscopic - Sun: corona

\section{Introduction}

The Solar Dynamics Observatory (SDO) Atmospheric Imaging Assembly (AIA, see Lemen et al. 2011) has been providing stunning broad-band extreme-ultraviolet (EUV) images of the Sun, since May 2010, revolutionizing our view of the solar corona. The AIA data are being used for a variety of purposes, however we believe that before any detailed quantitative work can be accomplished, the AIA calibration, the lines dominating the EUV bands, and the relevant atomic data need to be fully understood. This paper aims at clarifying a few relevant aspects, focusing mainly on the importance of $\operatorname{cool}(\log T[\mathrm{~K}]=5.0-5.8)$ transition-region (TR) lines in all the EUV bands. In O'Dwyer et al. (2010) we provided a preliminary description of the main spectral lines that are expected to dominate the AIA EUV bands for averaged regions (a coronal hole, quiet Sun, an active region and an M2-class flare). These results were not exhaustive of the many source regions which are present in the solar corona, and did not address the issue of the cool emission.

The presence of cool emission in the SDO/AIA bands is known, but has not been properly taken into account or quantified in detail previously. The AIA responses have only just recently been published (Boerner et al. 2011). It is very important to understand this cool emission contribution in order to ensure that the correct conclusions are reached when using AIA observations. The issues discussed in this paper are likely to affect several published results. For example, various authors (see, e.g. Schmelz et al. 2010, 2011; Aschwanden \& Boerner 2011) have recently used AIA EUV data to infer the thermal characteristics of coronal "warm" (1 MK) loops. In this paper, we provide examples where we make clear that AIA EUV data alone

\footnotetext{
* Appendices $\mathrm{A}-\mathrm{C}$ are available in electronic form at http://www . aanda.org
}

do not provide reliable information on the thermal characteristics of these loops. This is partly due to the contribution of cool emission in many AIA bands, for which very little atomic data are available, and in part probably due to the multi-thermal nature of the AIA bands. The fact that the AIA bands are sensitive to emission formed over a broad range of temperatures means that extreme care must be exercised when comparing features seen in different bands. This is especially true in active regions, where SOHO CDS observations have clearly shown that most warm loops at each location are almost isothermal in their crosssection (Del Zanna 2003a; Del Zanna \& Mason 2003). This fact, combined with the fact that cooler and hotter loops are persistently intermingled (Del Zanna et al. 2006), means that often different loops appear superimposed along each line-of-sight.

Another example is the proposal by De Pontieu et al. (2011) that cool, chromospheric material is continuously being heated to coronal $(T>1 \mathrm{MK})$ temperatures, because the same features have been observed both in $\mathrm{H}-\alpha$ (with Hinode Solar Optical Telescope, SOT) and in the 304, 171 and $211 \AA$ SDO/AIA bands, which were assumed to be dominated by He II (50000 K), Fe IX $(0.8 \mathrm{MK})$ and Fe xIV (2 MK). This interpretation is of particular relevance for coronal heating. However, there are two problems associated with their interpretation.

First, as those authors show, within an hour-long observation (cf. their movies s2 and s3), only two clear upward-propagating brightenings were simultaneously observed in the 304, 171 and $211 \AA$ bands, near the foot-points of warm loops, with typical velocities of $75 \mathrm{~km} \mathrm{~s}^{-1}$ (cf. their Fig. 2). In most locations there was good correlation between the blue-wing of the hydrogen $\mathrm{H}-\alpha$ and the He II $304 \AA$ intensity, but very little correlation with the other "coronal" bands. Running difference images in the "coronal" 171 and $211 \AA$ bands do show upward-propagating disturbances most of the time, but these are typical for warm 
loops (cf. SOHO/EIT, Berghmans \& Clette 1999; and TRACE Schrijver et al. 1999).

Second, an enhancement in the AIA 171 and $211 \AA$ bands clearly indicates heating of chromospheric material, but not necessarily to coronal (above $1 \mathrm{MK}$ ) temperatures. Indeed, here we present quantitative evidence that significant enhancements near the footpoints of warm active region loops in the AIA 171 and $211 \AA$ A bands can be due to cool (below $1 \mathrm{MK}$ ) emission.

In order to show this, we present simultaneous SDO/AIA and Hinode/EIS EUV Imaging Spectrometer (EIS, see Culhane et al. 2007) observations of an active region (NOAA 11127 , on 2010 Nov 23). EIS observes two wavelength bands (SW:

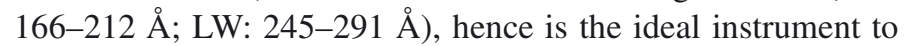
precisely show what emission is present in the AIA 171, 193, and $211 \AA$ bands because EIS actually observes nearly all wavelengths corresponding to these AIA bands. The EIS instrument also observes most ions contributing to the other AIA bands, hence can be used to estimate the detailed contribution to all the EUV bands, with the exception of the $304 \AA$ one.

As far as we are aware this is the first such detailed analysis presenting a direct comparison between Hinode/EIS and SDO/AIA. In this paper, we carry out a detail investigation of the contribution of cool emission to the SDO/AIA channels. The AIA response functions are discussed in Sect. 2. In Sect. 3, we select a cool footpoint region in an active region and perform inverse and forward-modelling using simultaneous EIS and SDO/AIA observations, to show which lines dominate the AIA count rates. We also study the temperature distribution of a warm loop leg, deriving the differential emission measure (DEM $(T)$ ) from the Hinode/EIS observations (having subtracted the background emission). In Sect. 4 we attempt to derive emission measures from the AIA observations for both the cool footpoint region and the loop leg. In Sect. 5 we draw our conclusions.

\section{The AIA response functions and associated atomic data}

Throughout this paper, we use the AIA responses calculated with the use of the CHIANTI ISOTHERMAL procedure, as outlined in the Appendix. We have then compared the results obtained using the same set of parameters as those adopted for the default AIA response functions which are available within Solarsoft, namely CHIANTI v.6 (Dere et al. 2009) atomic data and ionization tables, a constant pressure of $10^{15} \mathrm{~cm}^{-3} \mathrm{~K}$ and a set of "coronal" elemental abundances. The results are displayed as solid lines in Fig. 1. The response curves are very wide in temperature, and most of them are double-peaked, showing the multi-thermal nature of these bands. The default AIA response functions available within Solarsoft have been calculated with AIA Solarsoft programs. We found some small differences with the results obtained using these Solarsoft programs (displayed as dashed lines in the same figure), which led to the discovery (in collaboration with P. Boerner from the SDO/AIA team) of a software bug in their continuum calculation. As shown in Fig. 1, this bug only affected the responses at some temperatures, and the difference between the corrected and un-corrected curves is well within uncertainties. In fact, the overall accuracy of the preflight AIA calibration is estimated to be of order $25 \%$ (Boerner et al. 2011). To this, one would have to add an uncertainty in the emissivities of the spectral lines, which is difficult to assess, but would be at best of the order of 10-20\%. Indeed, benchmark studies of the most accurate atomic data in the EUV typically indicates, for
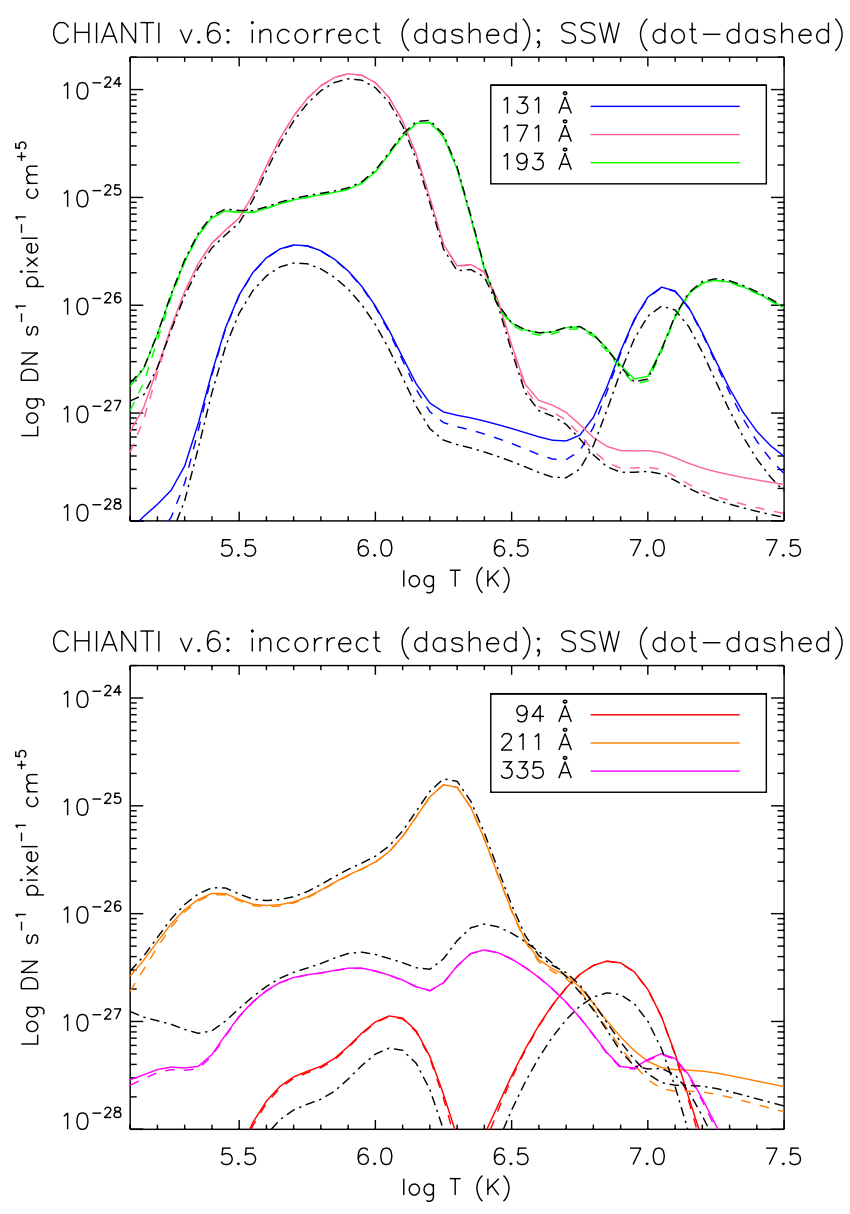

Fig. 1. The SDO AIA response functions calculated with the CHIANTI v.6 ion abundances, "coronal" abundances and constant pressure $\left(10^{15} \mathrm{~cm}^{-3} \mathrm{~K}\right)$, calculated with the correct program (solid lines) and the incorrect one (dashed lines). The default values available within SSW are shown as dot-dashed lines.

the strongest lines, this level of agreement. Another additional uncertainty is due to the lack of atomic data, which for some AIA spectral bands and temperatures is shown in this paper to amount to a factor of two.

The default responses available within Solarsoft are also shown in Fig. 1 (dot-dashed lines). They are slightly different because some correction factors to emission line emissivities are applied within the AIA Solarsoft programs.

The responses depend directly on the measured effective areas (Boerner et al. 2011), and the atomic data. The new ion abundances (Dere et al. 2009) represent a significant improvement over previous ones, and are used here. Large uncertainties are associated with the theoretical estimates of line emissivities, in particular those of the Li-like and Na-like ions, as described in Del Zanna et al. (2002).

Throughout this paper, we have improved the CHIANTI v.6 data by adopting new atomic data for Fe viI (Witthoeft \& Badnell 2008; Del Zanna 2009a), Fe vIII (Del Zanna 2009b), Fe xi (Del Zanna et al. 2010; Del Zanna 2010), and Fe xiv (Liang et al. 2010). These data will be available soon in the next CHIANTI release (Landi et al. 2011, version 7) with the exception of Fe vII, because of uncertainties in the identifications of the strongest lines (Young \& Landi 2009). These new data represent a significant improvement over previous ones, when individual emission lines are considered, however they provide overall minor 


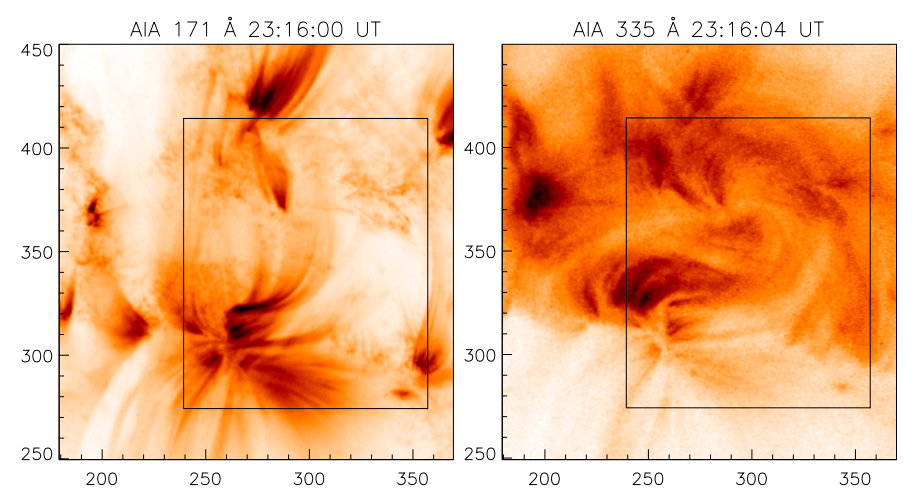

Fig. 2. SDO/AIA images of NOAA 11127 on 2010 Nov. 23 (left: $171 \AA$, right: $335 \AA$ ). The axes indicate arcseconds from Sun centre, towards the west and north. The boxes indicate the field-of-view of the Hinode/EIS observation in Fig. 3.

differences in the AIA responses (see Fig. A.1 in the Appendix). Significant differences in the responses are however found which depend on the choice of chemical abundances, as described in the Appendix. It is therefore recommended that appropriate abundances are used when calculating AIA responses. Some small differences in the AIA response functions are also obtained with different choices of density vs. temperature along the line of sight, hence it is recommended that users create their own AIA responses, which are best suited to each particular observation, following a simple procedure outlined in the Appendix. In this paper, we use the "photospheric" abundances of Asplund et al. (2009), because they better represent the oxygen/magnesium ratio observed by Hinode/EIS (see below).

\section{Observations and analysis}

Figure 2 shows AIA images of NOAA 11127 on 2010 Nov. 23, with the field-of-view (FOV: $120^{\prime \prime} \times 160^{\prime \prime}$ ) of the Hinode/EIS observation (Atlas_60), which was obtained by rastering the EIS $2^{\prime \prime}$ slit (from west to east) 60 times by $\simeq 2$ " steps. With $60 \mathrm{~s}$ exposure time, the observation lasted $\simeq 60 \mathrm{~min}$. The EIS study was designed by us, in collaboration with Young and Warren, to obtain the full EIS spectral range needed for the present analysis.

The EIS data were processed in various steps. We adopted the EIS software and database available within SSW to find the location of the dust, the warm and hot pixels. We then used standard SSW routines to locate the cosmic rays. We found that the amount of unusable pixels was so large that the line fitting methods failed at many locations. We therefore used custom-written software to linearly interpolate the unusable pixels, and visually inspect each single EIS exposure in all channels. We then used custom-written software to rotate each exposure, to correct for the slant of the spectra relative to the axes of the CCD (3.7 \pm 0.2 arcsec end-to-end), as found in Del Zanna \& Ishikawa (2009). The offset $\left(18^{\prime \prime}\right)$ between the SW and LW channels in the N-S direction was corrected, leaving a FOV of $120^{\prime \prime} \times 140^{\prime \prime}$.

The intensities of the EIS lines were obtained with Gaussian fitting (using custom-written software) of the spectra in data numbers, subtracting a constant bias. Figure 3 shows a selection of radiances, formed by (largely unblended) lines from ions (Fe vIII, Fe Ix, Fe x, Fe xII, Fe xIV, and Fe xvI) which are commonly expected to dominate the AIA 131, 171, 94, 193, 211, $335 \AA$ bands.
We then analysed the AIA full-disk data taken simultaneously with the EIS one-hour long observation. After processing the level-1 data with aia_prep v.4.0, we found that the location of the solar limb in the AIA images was not accurate, and we had to rescale the pixel sizes (nominally $0.6^{\prime \prime}$ ) by small amounts: $0.598^{\prime \prime}(304 \AA), 0.598^{\prime \prime}(94 \AA)$; 0.597" (131 ̊), 0.597" (335 $)$;

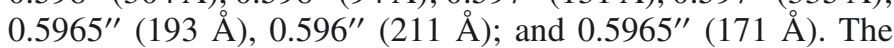
AIA instrument consists of four telescopes, where sections of the mirrors have been coated with different multilayers. The $304 \AA$ and $94 \AA$ bands share the same telescope, as is the case for the $131 \AA$ and $335 \AA$ bands, and the $193 \AA$ and $211 \AA$ bands. It is interesting to note that, within each telescope, the plate-scale seemed about the same. We checked the location of the limb in all ( $\simeq 2000)$ images. It appeared approximately (within $1^{\prime \prime}$ ) fixed, with some small jitter, probably due to thermal effects.

Given that the AIA spatial and temporal resolution are very different from the EIS ones, the AIA data have been carefully processed for a direct and meaningful AIA/EIS comparison. The characteristics of the EIS point-spread-function (PSF) are still not known, however the typical effective resolution is known to be about $3-4^{\prime \prime 1}$. This does not mean that the PSF of the EIS combined optics is $3-4^{\prime \prime}$. Indeed, there are at least two effects which degrade the EIS resolution. The first is the solar variability. It is clear, by inspecting the AIA images, that a small amount of variability is present at all times. The long exposures needed to obtain a good signal in the EIS spectra degrade its effective resolution. The second is the jitter of the Hinode spacecraft and the internal flexing of the EIS instrument, which combine to produce an effective random jitter of about $1-3^{\prime \prime}$ on very short (minutes) timescales. This occurs at all times and degrades the EIS resolution. In principle, with special observing sequences, it should be possible to accurately estimate the EIS PSF by comparison with AIA observatons. As explained in the Appendix, the data presented in this paper indicate good agreement between AIA and EIS PSF when the AIA images are convolved with a Gaussian of between 2 and 4 " full-width-half-maximum (FWHM). The results presented here are not significantly affected by the exact number for the PSF, and a value of $2^{\prime \prime}$ is chosen.

For each EIS slit position, we first convolved each AIA image. We then averaged those AIA images taken during each EIS exposure, rebinned them onto the "EIS pixel" size, and obtained a slice of the corresponding averaged AIA image. We then built a time-averaged "rebinned" image for direct comparison with the EIS monochromatic images.

The processed results are shown in Fig. 3. It is clear that the $131 \AA$ rebinned AIA image is in excellent agreement with the EIS Fe VIII monochromatic one, suggesting that this AIA band is dominated by this ion in this observation. The pointing of the EIS raster was obtained by cross-correlating the Fe vIII image with the AIA $131 \AA$ rebinned image. The co-alignment is very accurate $\left(1^{\prime \prime}\right)$ and indicates that the EIS mirror steps are such that successive EIS exposures are actually separated by $1.9^{\prime \prime}$ and not $2^{\prime \prime}$ as commonly thought. Figure B. 2 in the Appendix shows a good alignment in the features. A very similar number $\left(1.92^{\prime \prime}\right)$ was measured by Hara (2008).

There is generally good agreement between AIA and EIS count rates, indicating that: a) as a first approximation, an EIS Gaussian PSF of 2" FWHM seems appropriate; b) very low scattered light seems to be present in both AIA and EIS instruments. The EIS monochromatic images displayed in Fig. 3 clearly indicate that the core of the AR is dominated by 3-4 MK emission

\footnotetext{
${ }^{1}$ See the EIS wiki pages.
} 

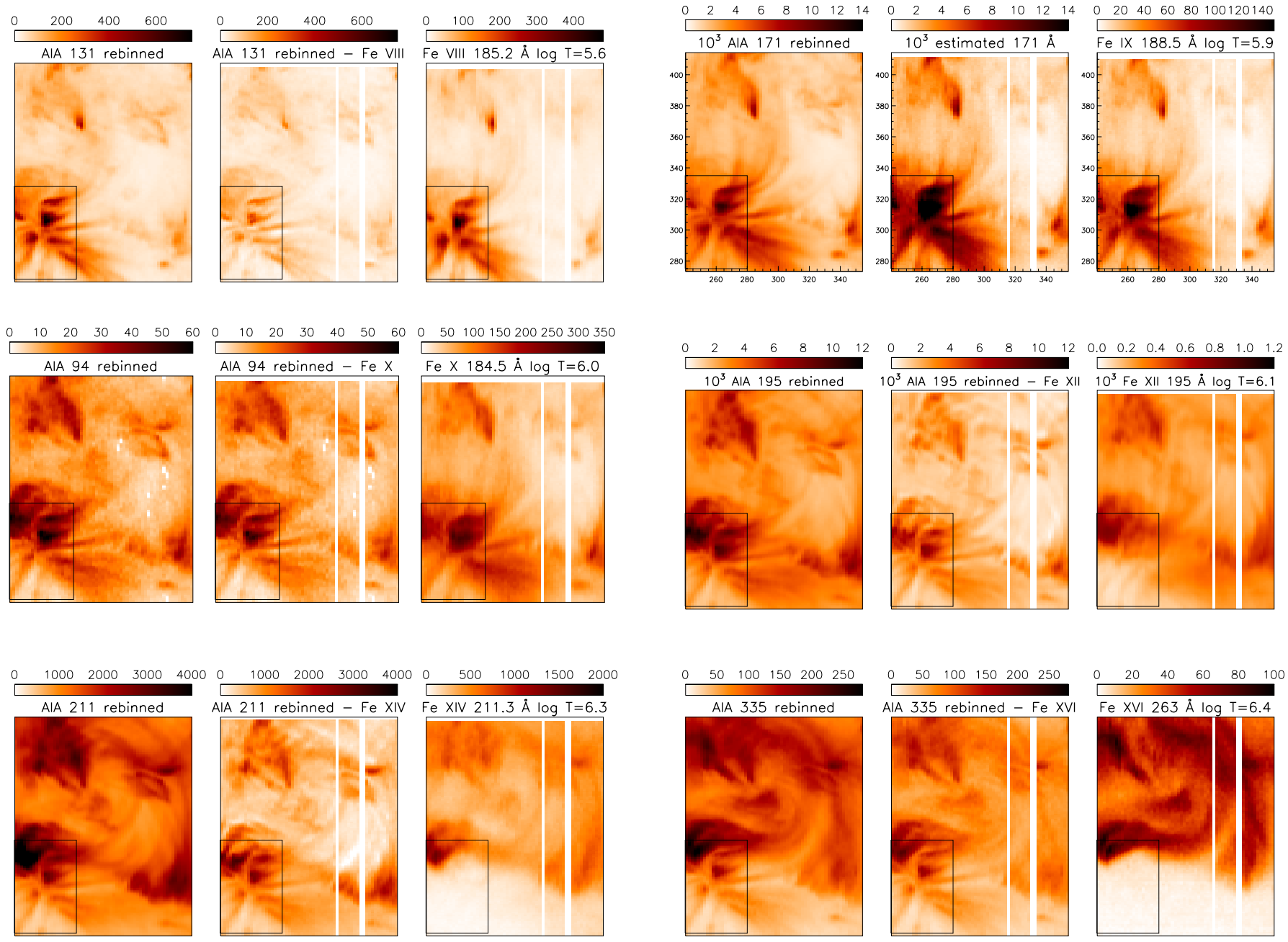

Fig. 3. For each band, the plots (negative, linear scale) show: (left) SDO/AIA images rebinned to the EIS resolution; (middle) AIA images with the contribution from the "dominant" ion subtracted (with the exception of the $171 \AA$ see text); (right) radiances in a selection of Hinode EIS lines, with the $\log T[\mathrm{~K}]$ of peak formation temperature (in equilibrium) indicated. The vertical blank lines are due to missing data. The colorbars for the EIS radiances indicate the actual calibrated units (phot $\mathrm{cm}^{-2} \mathrm{~s}^{-1} \operatorname{arcsec}^{-2}$ ), those in the other images DN s${ }^{-1}$ EIS pixel ${ }^{-1}$. The FOV of these images is 117.8 and 140.0 arcsec in the E-W and N-S direction. Their centers are 298.2, 344.3 arcsec from Sun center. The boxed areas indicate the FOV of Fig. 5, where strong cool emission is present.

(cf. Fe xIv, Fe xvI). In contrast, the southern part of the FOV is dominated by fans of cool/warm loops which are particularly strong in Fe vIII, Fe Ix. The legs of these warm loops are clearly observed in all AIA rebinned images, in spite of the fact that they do not emit at all in Fe xII, Fe xIv, and Fe xVI (as seen by EIS).

\subsection{Contributions to the AIA EUV bands}

To investigate further the emission line contributions to the AIA bands, we have subtracted, for each of the AIA images (Fig. 3), the contribution (DN/s) from the "dominant" ion, either directly observed with EIS, or estimated from the EIS radiances and the atomic data. The results are shown in Fig. 3. A few key aspects are discussed below, while more details are given later in the paper.

\subsection{1. $131 \AA$}

The AIA $131 \AA$ A band is dominated by two Fe vIII lines, at 130.94 , $131.24 \AA$. Their intensity has been estimated from the Fe vIII $185.2 \AA$ transition observed by Hinode/EIS, although this es- timate is quite uncertain, because of the temperature sensitivity of the soft X-ray vs. the EUV lines, and because of the uncertain atomic data for this ion (Del Zanna 2009b). The morphology of the AIA $131 \AA$ image, with the Fe vIII contribution subtracted, shows residual TR emission, due to ions formed at lower temperatures.

\subsection{2. $171 \AA$}

The AIA $171 \AA$ band is dominated by Fe Ix $171.0 \AA$. The line is very strong, but being at the edge of the EIS sensitivity, has very low count rates, and measurements are very uncertain (30-40\%). The morphology of the AIA $171 \AA$ is similar, but not the same as that for all the Fe Ix lines observed by EIS, as shown in Fig. 3, with the AIA $171 \AA$ image being closer to that for the lower- $T$ Fe vIII. The interpretation of this band is particularly complex, because of density and temperature effects. Towards the loop footpoints, the Fe IX $171.0 \AA$ is actually expected to have a lower emission, due to increasing densities. However, due to lowering temperatures, the Fe IX 171.0 $\AA$ emission should increase 

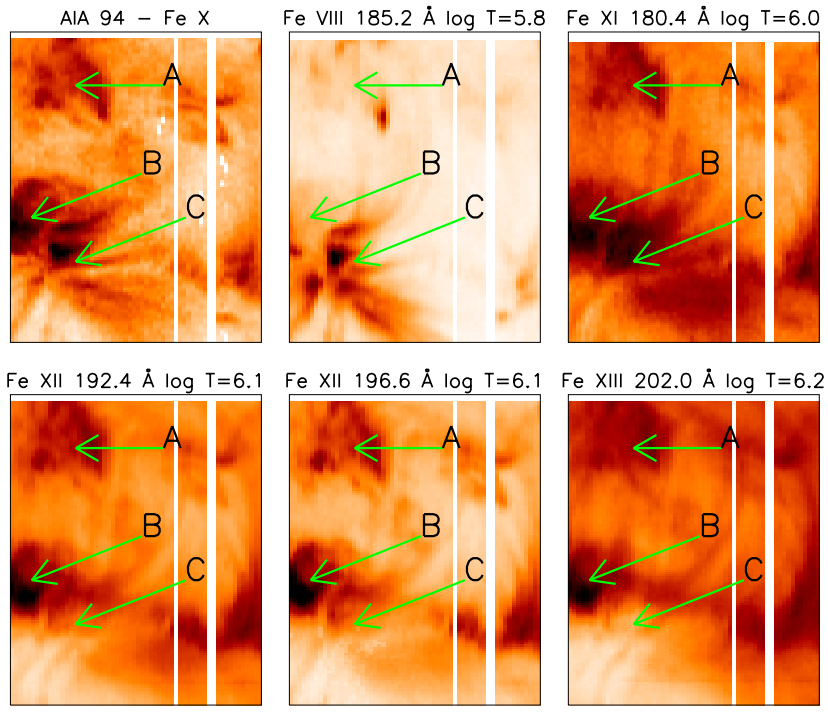

Fig. 4. The AIA $94 \AA$ A rebinned image with the estimated Fe x contribution removed (top left), together with a selection of EIS monochromatic images.

significantly (see Del Zanna 2009a). One puzzling aspect is the large AIA count rates due to Fe Ix $171.0 \AA$, as predicted from the Fe IX $188.5 \AA$ assuming a ratio (photons) of 19., the theoretical ratio at $\log T[\mathrm{~K}]=5.9$. Further detailed studies which take into account the temperature and density structure of the loops will be needed to find the reasons for the above discrepancies.

\subsection{3. $94 \AA$}

The AIA $94 \AA$ band has a significant contribution from Fe $x 94 \AA$. Its ratio with any of the EUV Fe x lines observed by EIS is strongly temperature-sensitive. For example, assuming a temperature of $1 \mathrm{MK}$, the ratio of the $94 \AA$ with the $184.5 \AA$ line is 0.028 , however at $\log T[\mathrm{~K}]=5.7$ it is predicted to be 0.011 . We know that the atomic data for the Fex $94 \AA$ line are not accurate, so the estimate is very uncertain. The AIA $94 \AA$ image, with the estimated Fe $\mathrm{x}$ contribution removed, clearly shows that the band is blended with lines formed over a range of temperatures. This is illustrated in Fig. 4, where the AIA $94 \AA$ rebinned image, with the estimated $\mathrm{Fe} x$ contribution removed, is shown together with a selection of EIS monochromatic images. There are at least two residual types of morphology: the first one is caused by emission in the 1.5-2 MK range, due to an ion formed at temperatures between Fe XI and Fe XIII (see region B in the figure). Inspection of region A in Fig. 4 suggests that the main blending line is most likely to be a decay to an excited state. As an example, Fig. 4 shows two transitions from Fe xII, one at $192.4 \AA$, which is a decay to the ground state, and one at $196.6 \AA$ which is a decay to an excited state, which is brighter when higher-densities are present. The second blend is clearly caused by cooler emission, due to ions with formation temperatures close to $\mathrm{Fe}$ VIII (see region $\mathrm{C}$ in the figure). This is not a surprise, since a number of strong unidentified transitions have been observed very close to $94 \AA$ in high-resolution solar spectra, well within the sensitivity of this AIA band.

\subsection{4. $193 \AA$}

The AIA/EIS comparison for this band is relatively straightforward, given that all the lines contributing to the AIA channel are easily observed with EIS. In average AR conditions, the dominant lines in the $193 \AA$ band are the three strong Fe XII 192.4, 193.5, 195.1 $\AA$. The contribution from these lines has been subtracted from the AIA rebinned image, and shown in Fig. 3. It is clear that significant residual cool TR emission is present.

\subsection{5. $211 \AA$}

In the cores of ARs, the dominant contribution to the $211 \AA$ band is a single Fe XIV transition at $211.3 \AA$, observed by Hinode/EIS (at the edge of the SW channel). However, the AIA $211 \AA$ band, with this Fe XIV contribution subtracted, shows dominant cool emission (Fig. 3) in many places.

\subsection{6. $335 \AA$}

Finally, in the case of the $335 \AA$ band, the dominant contribution in the core of active regions is from the Fe XVI $335.4 \AA$ transition, normally blended with $\mathrm{Mg}$ vIII and Fe XII. The ratio with the Fe XVI $263 \AA$, observed by EIS, is slightly temperature sensitive. The Fe XVI contribution to the AIA $335 \AA$ has been subtracted assuming a ratio of 17.5 (photons), and the residual (see Fig. 3) again clearly shows that the $335 \AA$ band is dominated by cool emission in many places.

\subsection{Discussion of cool emission: loop footpoint}

A lot of small-scale activity is present in all the AIA images, in particular close to the legs of the warm loops, and over timescales as short as the AIA cadence ( $12 \mathrm{~s}$, hence much shorter than the EIS exposure time). Upward-propagating features are seen in all EUV bands, with the exception of the $304 \AA$ band, where the opposite is quite often observed. As in the observations shown by De Pontieu et al. (2011) (cf. their movies s2 and s3), only occasionally upward-propagating features are simultaneously seen in the 304 and in the "coronal" 171,193 and $211 \AA$ bands. These events are not clearly observed in the other bands because of low signal-to-noise. The much lower spatiotemporal resolution of the EIS instrument does not allow a detailed characterisation of such single events, however EIS clearly observes ensembles of such events, near the footpoints of the warm loops, and indicates that a considerable amount of cool emission is present.

To quantify the contribution of cool lines to the AIA bands, we selected a region " $B$ " ( six EIS pixels) at the base of an ensemble of loops, indicated in Fig. 5. This region has strong $\mathrm{O} v$ emission which is not co-spatial with the emission at higher temperatures such as Fe vIII (see Del Zanna 2003a; and Young et al. 2007, for a description of how loop foot-points are visible at progressively different temperatures). Table 1 shows the observed AIA count rates in six of the seven AIA EUV bands, rebinned over the EIS resolution and averaged over region "B".

We then obtained an EIS averaged spectrum for region $\mathrm{B}$ and performed a DEM analysis on the EIS intensities, assuming a spline functional (see Del Zanna 1999, for details). The DEM (see Fig. 6) shows a significant peak at $\log T[\mathrm{~K}]=5.7$. Good agreement between the predicted and observed radiances in $\mathrm{O} v$, $\mathrm{O}$ vi, $\mathrm{Mg} \mathrm{v}$, and Mg vi lines was obtained adopting the "photo- 

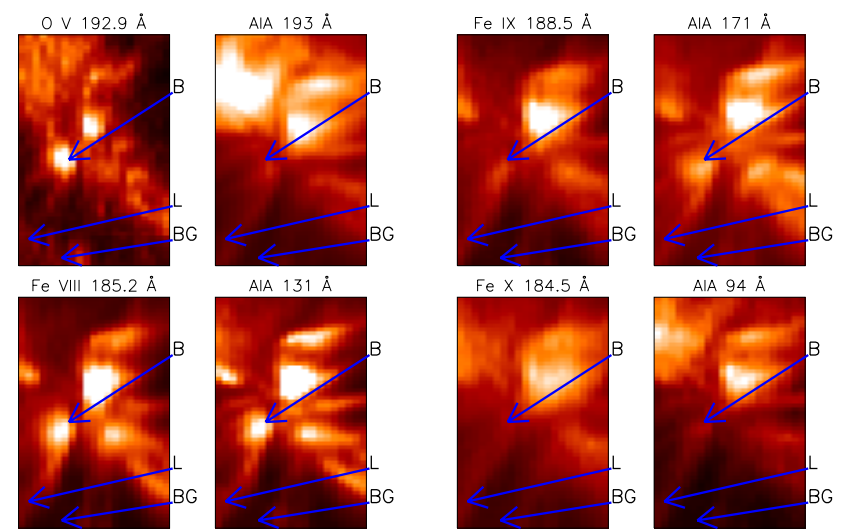

Fig. 5. Radiances in Hinode/EIS Ov, Fe vIII, Fe Ix and Fe $x$ lines. The SDO/AIA images have been rebinned onto the EIS resolution for the 193, 131, 171, and $94 \AA$ bands. The location of a loop base "B", leg "L" and relative background "BG" are indicated. The field of view is within solar $X=240-280$ and solar $Y=275-335$ arcsec from Sun centre.

Table 1. Observed and simulated count rates for the SDO/AIA channels for region "B".

\begin{tabular}{lccc}
\hline \hline Band $(\AA)$ & Obs. (AIA) & Pred. (EIS) & Pred. (DEM) \\
\hline 94 & 22 & - & 11 \\
131 & 422 & - & 492 \\
171 & 7323 & 8910 & 12135 \\
193 & 4163 & 4826 & 3474 \\
211 & 1363 & 2104 & 802 \\
335 & 93 & - & 57 \\
\hline
\end{tabular}

Notes. Column 2 indicates the observed AIA count rates (averaged $\mathrm{DN} / \mathrm{s}$ per EIS pixel). Column 3 shows the simulated AIA count rates, obtained directly from the EIS spectra. Column 3 shows the simulated AIA count rates, obtained from the DEM modeling.

spheric" abundances of Asplund et al. (2009). This suggests that, at least near the footpoint, the observed loop structure does not have a first ionization potential (FIP) bias, given that oxygen is a high-FIP and Mg a low-FIP element. This result is in agreement with those obtained from neon and magnesium lines in warm loops (Del Zanna 2003b; Del Zanna \& Mason 2003).

We then used this DEM to forward-model and simulate the AIA count rates, using the same set of parameters adopted for the inversion. This was done by calculating line and continuum emissivities as a function of wavelength and temperature. These were then folded with the AIA effective areas and the DEM distribution to obtain AIA simulated count rates as a function of wavelength and temperature. These count rates were then summed over wavelength, to obtain the AIA simulated count rates as a function of temperature, shown in Fig. 7.

We also integrated the AIA simulated count rates over temperature, and produced simulated AIA count rates as a function of wavelength, to be compared to those obtained directly from the EIS spectrum. A bin size of $0.022 \AA$ and a $F W H M$ of $0.08 \AA$ was adopted. For the 131, 171, 94, 193, 211 and $335 \AA$ channels the resulting simulated spectra are displayed in Figs. 8, 9, $11,12-14$ respectively. The main lines in the spectra are labeled. The total AIA simulated count rates are displayed in Table 1 (Col. 4).

We then converted the EIS radiances into simulated AIA count rates, for the 171, 193 and $211 \AA$ channels, using the EIS (Lang et al. 2006) and AIA (Boerner et al. 2011) effective areas (as currently available within Solarsoft). The results are shown in

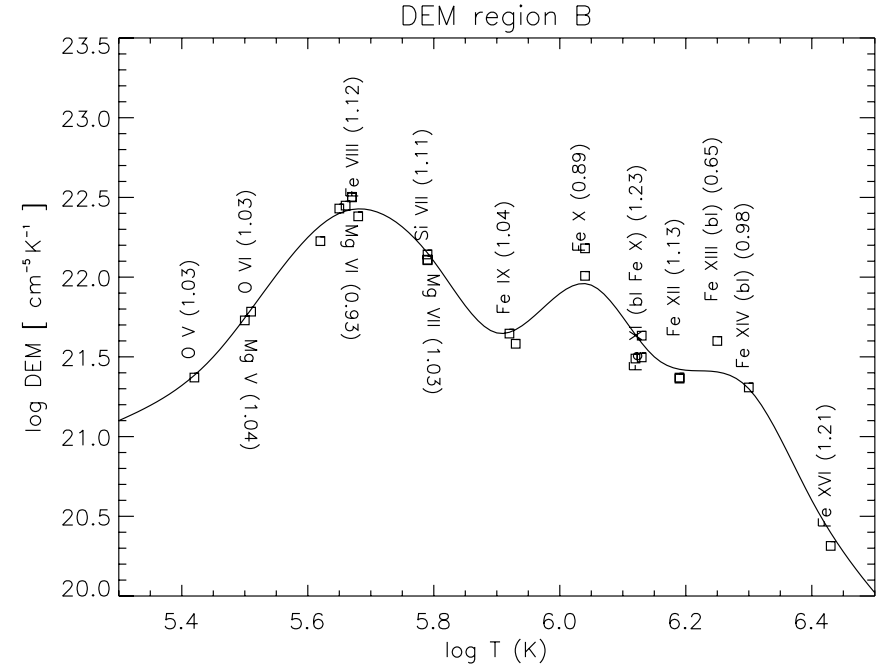

Fig. 6. The DEM of the loop foot-point region "B", derived from Hinode/EIS. The numbers in parentheses are the theoretical vs. the observed intensity ratio. The points are plotted at the temperature of maximum ion abundance in equilibrium, and at the theoretical vs. the observed intensity ratio multiplied by the DEM value.
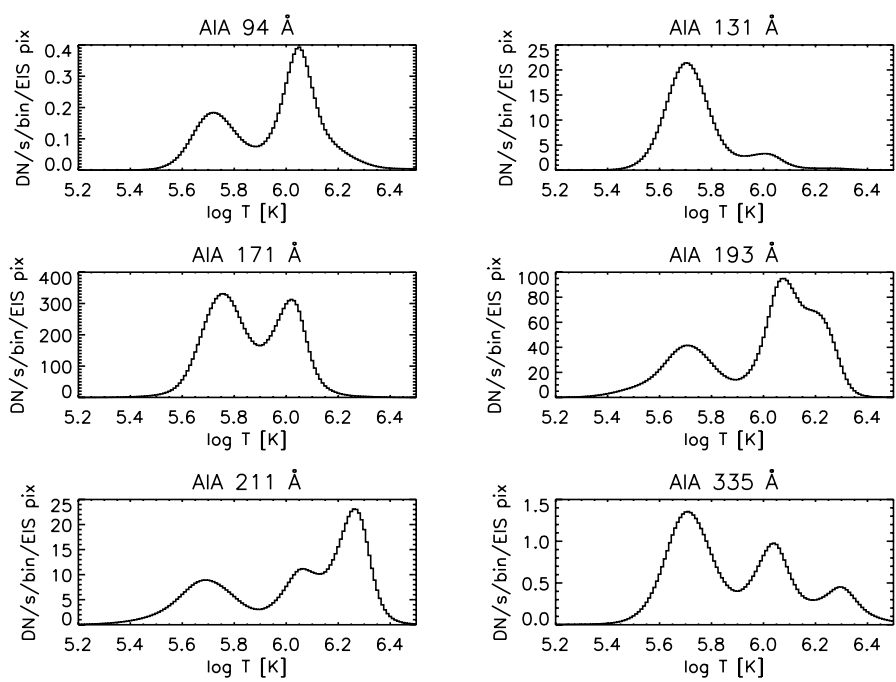

Fig. 7. Simulated AIA count rates as a function of temperature for region $\mathrm{B}$.

Figs. 9, 12, and 13. The prominent lines (for region B) contributing to the AIA 193 and $211 \AA$ count rates are listed in Tables 2 and 3 . In these tables, we have indicated the approximate identification. We used the detailed results from Del Zanna (2009a) to identify a feature as a cool TR line. At the bottom of the tables, a summary of the AIA count rates in terms of coronal and cool emission is given (the totals include weaker lines not listed in the Tables). The totals of the simulated AIA count rates are shown in Cols. 3, 4 of Table 1. Note that these numbers are higher than the totals in Tables 2 and 3, because they include a forest of weak lines.

\subsection{1. $131 \AA$}

As shown in Table 1, good agreement is found between observed and simulated AIA count rates for the $131 \AA$ band, dominated by Fe viII. As shown in Fig. 8, there are various $\mathrm{O}$ vi, $\mathrm{Ne}$ vi $\mathrm{Ne}$ VII 
Table 2. List of the main Hinode EIS spectral lines contributing to the SDO AIA $193 \AA$ channel in the loop footpoint region B.

\begin{tabular}{|c|c|c|c|c|}
\hline$\underline{\lambda_{\mathrm{o}}}$ & DN (EIS) & $R$ (EIS) & CR (AIA) & ID \\
\hline 184.54 & 3147 & 137 & 30 & Fe $X$ \\
\hline 185.24 & 7305 & 266 & 84 & Fe VIII (bl) \\
\hline 186.63 & 7880 & 209 & 143 & Fe VIII (bl) \\
\hline 186.88 & 2666 & 67 & 52 & Fe XII (2) (bl) \\
\hline 187.27 & 974 & 23 & 21 & Fe VIII \\
\hline 187.97 & 842 & 17 & 22 & $\mathrm{u}$ \\
\hline 188.22 & 8545 & 168 & 232 & $\mathrm{Fe} X \mathrm{XI}$ \\
\hline 188.31 & 4466 & 86 & 123 & Fe XI \\
\hline 188.45 & 1098 & 21 & 31 & Fe VII (bl) \\
\hline 188.51 & 4070 & 76 & 116 & Fe IX (bl ?) \\
\hline 188.64 & 763 & 14 & 22 & $\mathrm{u}$ \\
\hline 188.82 & 607 & 11 & 18 & $\mathrm{u}$ \\
\hline 189.01 & 555 & 10 & 17 & Fe XI \\
\hline 189.12 & 493 & 8 & 15 & $\mathrm{Fe} X \mathrm{XI}$ \\
\hline 189.37 & 484 & 8 & 16 & $\mathrm{u}(\mathrm{VII})$ \\
\hline 189.49 & 738 & 12 & 24 & Fe VII \\
\hline 189.60 & 447 & 7 & 15 & $\mathrm{u}$ \\
\hline 189.73 & 516 & 8 & 18 & Fe XI \\
\hline 189.95 & 3410 & 52 & 119 & Fe IX \\
\hline 190.05 & 4027 & 60 & 141 & Fe X (bl) \\
\hline 190.91 & 486 & 7 & 18 & $\mathrm{u}(\mathrm{bl}$ TR) \\
\hline 191.05 & 482 & 6 & 18 & Fe XII (bl ?) \\
\hline 191.23 & 2186 & 28 & 84 & Fe IX (bl) \\
\hline 191.42 & 449 & 6 & 17 & $\mathrm{u}$ \\
\hline 191.61 & 867 & 11 & 34 & Mn IX? \\
\hline 191.72 & 446 & 6 & 18 & u (TR) \\
\hline 192.03 & 1715 & 21 & 68 & Fe XI (bl Fe VIII) \\
\hline 192.11 & 1268 & 15 & 51 & u (TR) \\
\hline 192.20 & 734 & 9 & 29 & $\mathrm{u}(\mathrm{X})$ \\
\hline 192.30 & 695 & 8 & 28 & $\mathrm{u}$ \\
\hline 192.39 & 4358 & 51 & 174 & Fe XII \\
\hline 192.64 & 1965 & 22 & 79 & $\mathrm{u}(\mathrm{bl} \mathrm{Fe} \mathrm{XI)}$ \\
\hline 192.81 & 4901 & 55 & 195 & $\mathrm{Fe}$ XI (bl O V) \\
\hline 192.94 & 2406 & 27 & 96 & $\mathrm{O} \mathrm{V}$ \\
\hline 193.15 & 292 & 3 & 11 & u (TR) \\
\hline 193.29 & 846 & 9 & 33 & $\mathrm{u}(\mathrm{TR})$ \\
\hline 193.51 & 10211 & 109 & 389 & Fe XII \\
\hline 193.72 & 2051 & 22 & 76 & $\mathrm{Fe} X$ \\
\hline 193.87 & 350 & 4 & 13 & $\mathrm{u}$ \\
\hline 193.99 & 1117 & 12 & 40 & Fe VIII \\
\hline 194.11 & 445 & 5 & 15 & u (TR) \\
\hline 194.32 & 673 & 7 & 22 & $\mathrm{u}(\mathrm{TR})$ \\
\hline 194.69 & 7581 & 76 & 214 & Fe VIII \\
\hline 195.12 & 20419 & 203 & 454 & Fe XII (2) \\
\hline 195.42 & 4455 & 44 & 81 & $\mathrm{u}(\mathrm{TR}, \mathrm{VIII})$ \\
\hline 195.51 & 2672 & 26 & 45 & $\mathrm{u}$ \\
\hline 195.76 & 764 & 8 & 11 & $\mathrm{u}$ \\
\hline 196.00 & 6279 & 62 & 71 & Fe VIII (bl) \\
\hline 196.09 & 1854 & 18 & 19 & Fe VII \\
\hline 196.25 & 2602 & 26 & 24 & Fe VII (sbl) \\
\hline 196.66 & 2574 & 26 & 18 & Fe XII \\
\hline 197.87 & 4463 & 50 & 19 & Fe IX \\
\hline \multirow[t]{5}{*}{198.56} & 2919 & 36 & 10 & S VIII (bl) \\
\hline & Totals & & 300 & $\mathrm{u}(\mathrm{TR})$ \\
\hline & & & 1285 & TR \\
\hline & & & 365 & $\mathrm{u}$ (Coronal) \\
\hline & & & 1960 & Coronal \\
\hline
\end{tabular}

Notes. $\lambda_{0}(\AA)$ is the measured wavelength, DN (EIS) are the total EIS data numbers in each line, $R$ (EIS) are the EIS radiances in phot $\mathrm{cm}^{-2} \mathrm{~s}^{-1} \operatorname{arcsec}^{-2}$, CR (AIA) are the contribution to the AIA band as DN/s per EIS pixel. The column ID provides the identification (bl: blended; sbl: self-blend; u: unidentified; TR: transition-region line). When known, the class of a line is given (i.e.: u VII is a line with a morphology similar to Fe VII).

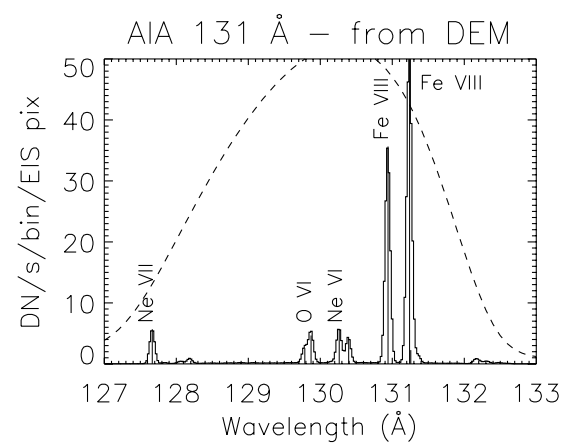

Fig. 8. AIA $131 \AA$ A simulated spectrum from the DEM modeling for region $\mathrm{B}$.

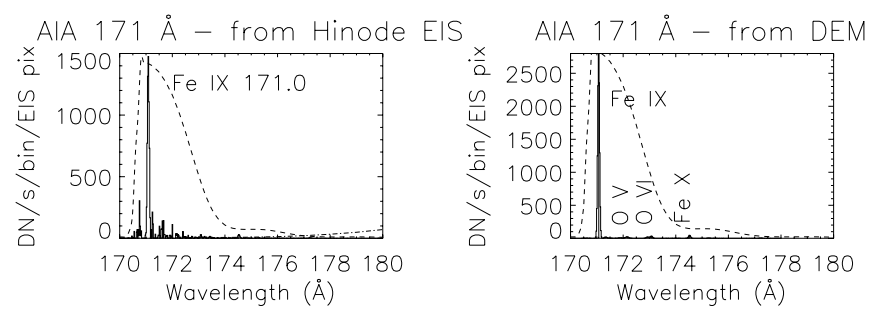

Fig. 9. AIA $171 \AA$ A simulated spectra from the EIS observation and the DEM modeling for region $\mathrm{B}$.

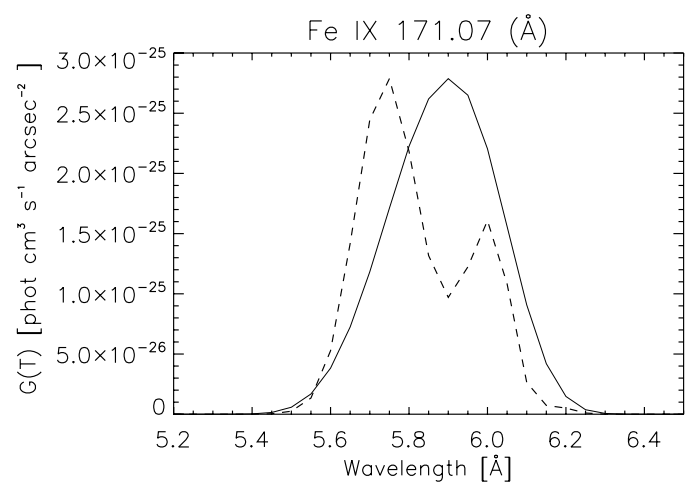

Fig. 10. The contribution function $G(T)$ for the Fe Ix $171 \AA$ line (solid line), with the product $\operatorname{DEM}(T) \times G(T)$ (dashed line), normalised.

TR lines which are obvious candidates to explain the residual TR emission in the $131 \AA$ band, shown in Fig. 3. Obviously, if $10 \mathrm{MK}$ plasma is present, other transitions due to Fe xx, Fe xxI and Fe XXIII become important for this band.

\subsection{2. $171 \AA$}

The estimate of what the AIA count rates should be, based on the EIS observation (Table 1), is uncertain, given the large sensitivity to where the true bias of the EIS CCDs lies. Despite the low signal, no significant $\mathrm{O} v$ and $\mathrm{O} v \mathrm{v}$ emission is observed by EIS, as shown in Fig. 9, so the emission is dominated by the Fe IX $171 \AA$ line. This is confirmed by the DEM modeling: despite significant emission measures at lower and other temperatures, the DEM predicts negligible contribution to this AIA band from the O v $172.2 \AA$ and O vi $173.0 \AA$ self-blend, in agreement with the suggestion given by De Pontieu et al. (2011).

One aspect which has however been overlooked in the work by De Pontieu et al. (2011) is the fact that the Fe Ix $171 \AA$ 


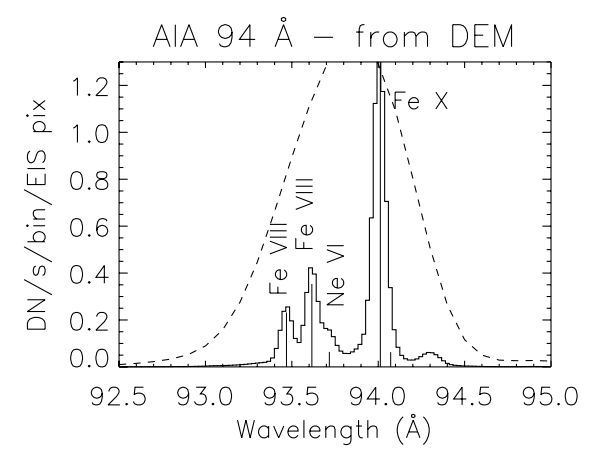

Fig. 11. AIA $94 \AA$ simulated spectrum from the DEM modeling for region $\mathrm{B}$.

is sensitive to very cool, down to $\log T[\mathrm{~K}]=5.5$, emission, as shown in Fig. 10. Figure 10 shows the $G(T)$ for Fe IX $171.0 \AA$ and the product of $\operatorname{DEM}(T) \times G(T)(\operatorname{DEM}(T)$ corresponds to the DEM curve in Fig. 6). It can be seen that $\operatorname{DEM}(T) \times G(T)$ has a peak at $\log T[\mathrm{~K}]=5.75$. Figure 7 shows that a significant part of the observed count rates in region $\mathrm{B}$ originates from plasma at $\log T[\mathrm{~K}]=5.7$, due almost entirely to Fe IX.

\subsection{3. $94 \AA$}

For the $94 \AA$ channel, the DEM modeling shows a significant contribution from Fe vIII 7f-3d transitions (see Fig. 11), for which atomic data are very uncertain. Work is in progress on a new calculation, but we anticipate here that the current emissivities are underestimated. As shown in Fig. 11, even with the current atomic data, the contribution from these Fe viII cool lines is significant, hence they can at least in part be responsible for the cool TR emission present in the AIA band (as shown in Fig. 3 and discussed previously).

The AIA count rates for the $94 \AA$ channel are underpredicted by a factor of two, which is partly due to the very approximate atomic data for the Fe vIII $7 f-3 d$ and Fe $x$ 4s-3p lines, and partly due to blending with other transitions. Obviously, if much hotter emission is present, Fe XVIII becomes dominant in this band.

\subsection{4. $193 \AA$}

The spectrum of region B shown in Fig. 12, clearly indicates which lines contribute significantly to the residual cool TR emission in the AIA $193 \AA$ band (in Fig. 3). Very good agreement (within a relative $16 \%$ ) between the AIA observed count rates and those predicted directly from the EIS spectrum is found (see Table 1). This is almost independent from the choice of EIS CCD bias, and suggests a good relative calibration between these two channels.

The new atomic data (Fe vII, Fe vIII, Fe XI) are important for this band. The DEM modelling fails to reproduce the Fe VII, which are underestimated by a factor of about four. The DEM modelling predicts AIA count rates close to but below those observed. This is partly caused by a large number of unidentified lines (see Table 2), some of which are known for sure to be cool lines, as described in Del Zanna (2009a). Overall, even for this band, a significant contribution from lines formed below $1 \mathrm{MK}$ is present, as can be seen in Fig. 7.
Table 3. List of the main Hinode EIS spectral lines contributing to the SDO AIA $211 \AA$ A channel, as in Table 2 .

\begin{tabular}{|c|c|c|c|c|}
\hline$\lambda_{\mathrm{o}}$ & DN (EIS) & $R(\mathrm{EIS})$ & CR (AIA) & ID \\
\hline 202.04 & 2303 & 87 & 25 & Fe XIII (bl) \\
\hline 202.42 & 653 & 29 & 10 & Fe XI \\
\hline 202.86 & 916 & 48 & 19 & $\mathrm{u}$ (VII-VIII) \\
\hline 203.72 & 410 & 29 & 16 & Fe XII (bl) \\
\hline 203.83 & 1005 & 72 & 41 & Fe XIII (2) \\
\hline 204.73 & 540 & 49 & 38 & $\mathrm{Fe}$ VIII \\
\hline 204.92 & 180 & 17 & 14 & Fe XIII (+ Fe XI) \\
\hline 205.06 & 316 & 31 & 26 & Cr VIII (?) \\
\hline 205.72 & 127 & 14 & 14 & Cr VIII (?) \\
\hline 206.17 & 114 & 14 & 16 & $\mathrm{u}(\mathrm{TR})$ \\
\hline 206.25 & 88 & 11 & 13 & $\mathrm{u}(\mathrm{TR})$ \\
\hline 206.33 & 89 & 11 & 13 & Fe XII (bl) \\
\hline 206.78 & 132 & 18 & 24 & $\mathrm{Fe} \mathrm{VII}$ \\
\hline 206.96 & 52 & 7 & 10 & $\mathrm{u}$ \\
\hline 207.15 & 348 & 51 & 72 & Cr VIII (?) \\
\hline 207.24 & 99 & 15 & 21 & $\mathrm{u}(\mathrm{TR})$ \\
\hline 207.45 & 283 & 44 & 66 & $\mathrm{u}(\mathrm{bl} \mathrm{Fe} \mathrm{X)}$ \\
\hline 207.75 & 184 & 30 & 48 & $\mathrm{u}(\mathrm{TR})$ \\
\hline 207.94 & 126 & 21 & 35 & $\mathrm{u}(\mathrm{TR})$ \\
\hline 208.41 & 29 & 5 & 10 & $\mathrm{u}$ \\
\hline 208.54 & 39 & 7 & 14 & ? Ca XVI (bl Ca XV) \\
\hline 208.62 & 28 & 5 & 10 & Cr VIII (?) \\
\hline 208.69 & 106 & 20 & 39 & ? Ca XV (bl Ca XVI) \\
\hline 208.85 & 124 & 25 & 48 & $\mathrm{Fe} \mathrm{VII}$ \\
\hline 209.00 & 39 & 8 & 16 & $\mathrm{u}(\mathrm{TR})$ \\
\hline 209.10 & 34 & 7 & 14 & $\mathrm{u}(\mathrm{TR})$ \\
\hline 209.45 & 136 & 30 & 63 & $\mathrm{u}(\mathrm{TR})$ \\
\hline 209.55 & 54 & 12 & 26 & $\mathrm{u}$ \\
\hline 209.64 & 92 & 21 & 45 & u (TR, bl Fe XIII) \\
\hline 209.76 & 100 & 24 & 51 & Fe VII \\
\hline 209.94 & 140 & 34 & 75 & u (TR, bl Fe XIII) \\
\hline 210.16 & 37 & 9 & 21 & $\mathrm{u}$ \\
\hline 210.44 & 49 & 13 & 29 & $\mathrm{u}$ \\
\hline 210.65 & 80 & 22 & 50 & $\mathrm{u}$ \\
\hline 210.95 & 22 & 6 & 14 & $\mathrm{u}$ \\
\hline 211.20 & 20 & 6 & 13 & $\mathrm{u}$ \\
\hline 211.32 & 248 & 77 & 163 & Fe XIV (bl) \\
\hline 211.45 & 51 & 16 & 34 & $\mathrm{u}$ \\
\hline 211.72 & 48 & 16 & 31 & Fe XII (bl?) \\
\hline \multirow{4}{*}{\multicolumn{3}{|c|}{ Totals }} & 380 & $\mathrm{u}(\mathrm{TR})$ \\
\hline & & & 290 & $\mathrm{TR}$ \\
\hline & & & 290 & $\mathrm{u}$ (Coronal) \\
\hline & & & 409 & Coronal \\
\hline
\end{tabular}

\subsection{5. $211 \AA$}

Figure 13 clearly shows that most of the lines contributing to the $211 \AA$ band are observed by EIS, given the sharp decline of the AIA effective area above $212 \AA$. In spite of this, the total AIA count rates predicted from the EIS spectrum are $50 \%$ higher than the observed ones. There is a $20 \%$ or so uncertainty due to a forest of weak lines and the location of the EIS CCD bias, however the discrepancy is present and suggests a calibration problem with either EIS, AIA or both.

More than $50 \%$ of the observed spectral lines are unidentified. The most prominent ones are labeled in Fig. 13, and detailed in Table 3. Indeed, if one considers only the strongest lines, about half are due to unidentified lines, for which no atomic data are yet available. The strongest lines have been observed since the early EUV rocket flights in the 1960's. 

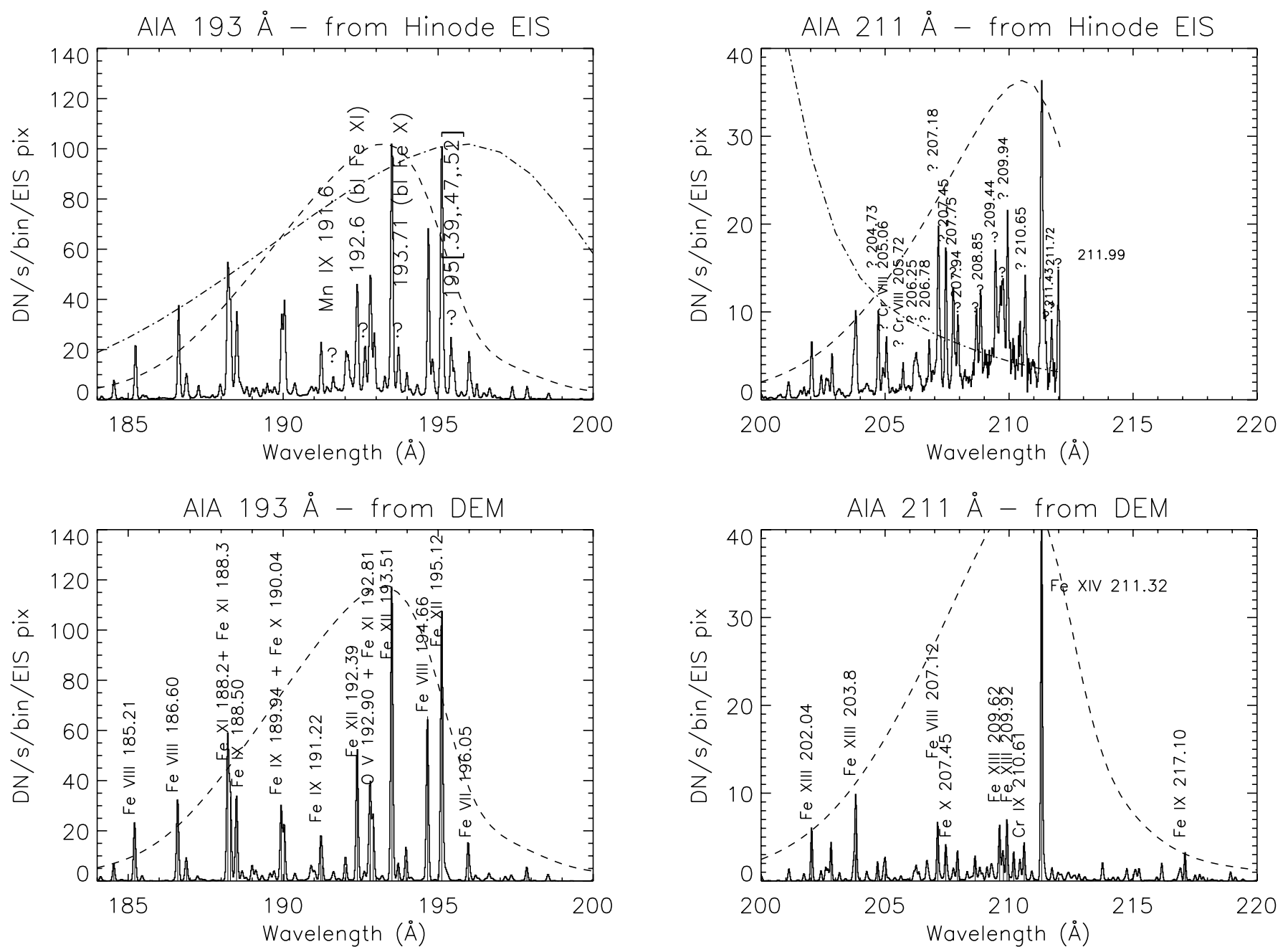

Fig. 12. Simulated AIA $193 \AA$ count rates for region "B", from the observed EIS spectrum (top) and from the DEM modeling (bottom). The dashed and dot-dash curves are the normalised AIA and EIS effective areas respectively. The strongest unidentified lines are labeled (top).

Another $20 \%$ of the observed spectral lines are clearly due, as detailed in Del Zanna (2009a), to TR lines, for which atomic data are either unavailable or inaccurate. The few lines for which we have atomic data already produce a significant lowtemperature contribution, as shown in Fig. 7. The spectral region above $212 \AA$, not observed by EIS, is also a region which has received very little attention to date, and it is likely that a significant contribution from unidentified lines is also missing there. In summary, the total AIA count rates (Table 1) are underpredicted by more than a factor of two due to a lack of atomic data.

\subsection{6. $335 \AA$}

The $335 \AA$ band is clearly sensitive to a host of cool transitions in the 310-350 ̊ range (see Fig. 14). A significant contribution (about $50 \%$ ) is predicted to come from lower wavelengths, around 184 and $131 \AA$. The first is due to a predicted secondorder peak in the mirror reflectivity around $184 \AA$ (Boerner et al. 2011), while the second is due to cross-talk.

The $131 \AA$ and $335 \AA$ bands share the same telescope, and both channels are illuminated at all times. As the focal-plane

Fig. 13. Simulated AIA $211 \AA$ A count rates for region "B", from the observed EIS spectrum (top) and from the DEM modeling (bottom) as in Fig. 12.

filter does not reject all light from the opposite channel, there is some cross-talk between the two wavelength channels. The presence of the cross-talk was known (Boerner et al. 2011), and indeed the AIA effective area for the $335 \AA$ channel includes an estimate of the contribution from the $131 \AA$ band (as it does for the second-order contribution).

The cross-talk was predicted by Boerner et al. (2011) to be significant only in flaring conditions, however in the simulation presented here it appears to be significant even in normal active region conditions. The reasonable agreement between the observed and simulated count rates supports the validity of the estimated cross-talk and second-order peak. It is also possible that some contribution from He II $304 \AA$ exists. Overall, cool emission for region "B" dominates this band, as shown in Fig. 7.

\subsection{The warm loop leg}

To test how accurately the thermal distribution of warm loops can be inferred from SDO/AIA observations, we have selected a loop leg region "L", shown in Fig. 5, and a nearby background/foreground region "BG". Region "L" has strong emission just below $1 \mathrm{MK}$. The AIA observed count rates, together 

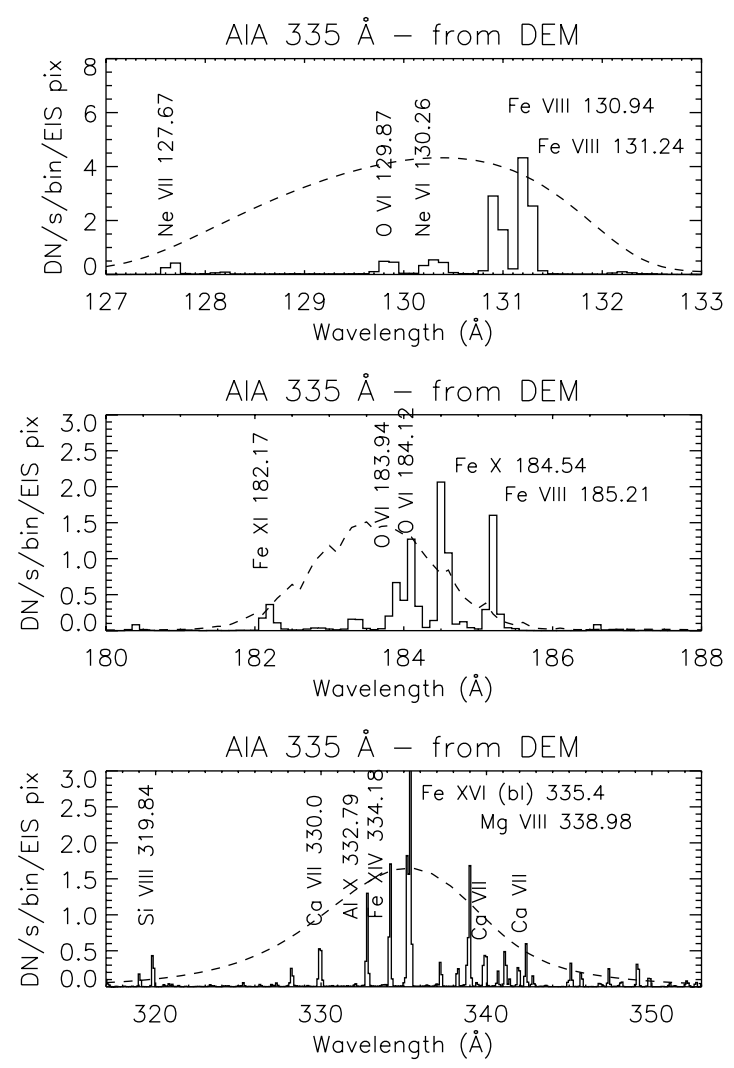

Fig. 14. AIA simulated spectra in the $335 \AA$ band from the DEM modeling for region $\mathrm{B}$, around $130 \AA$ (top), $184 \AA$ (middle) and $335 \AA$ (bottom).

Table 4. AIA count rates over the loop leg (L) and the background region $(\mathrm{BG})$.

\begin{tabular}{lcccccc}
\hline \hline$(\AA)$ & $L$ & BG & $L_{\text {bgs }}$ & $L($ EIS $)$ & BG (EIS) & Pred. $L_{\text {bgs }}$ \\
\hline 94 & 13.8 & 4.8 & 9 & - & - & 3 \\
131 & 162 & 60 & 102 & - & - & 103 \\
171 & 3705 & 2256 & 1449 & 9919 & 5004 & 4684 \\
193 & 2290 & 1469 & 821 & 2558 & 1611 & 631 \\
211 & 682 & 416 & 266 & 877 & 622 & 116 \\
335 & 41 & 22 & 19 & - & - & 14 \\
\hline
\end{tabular}

Notes. The AIA count rates are in averaged DN/s per EIS pixel. $L_{\mathrm{bgs}}$ are the background-subtracted AIA count rates. $L$ (EIS) and BG (EIS) are the count rates for the loop leg $(L)$ and background regions, directly based on the EIS spectra. Pred. $L_{\mathrm{bgs}}$ are the background-subtracted rates for the loop leg as predicted from the DEM modeling.

with those simulated (based on the Hinode EIS spectra) are displayed in Table 4.

We have followed the same analysis procedure as described previously for the loop base region "B", and obtained a set of background-subtracted Hinode EIS line radiances. The hottest lines with marginal residual counts are due to Fe XII.

The EM loci curves for a few strong lines are shown in Fig. 15 (top), indicating a near-isothermal plasma with $\log T[\mathrm{~K}]=5.95$, as confirmed by the DEM modelling, with results shown in Fig. 15 (bottom). For a description of the EM Loci method, first introduced by Strong (1978) and later applied by Del Zanna \& Mason (2003) and Del Zanna (2003b) to show that warm AR loops are close to isothermal, see Del Zanna et al. (2002).
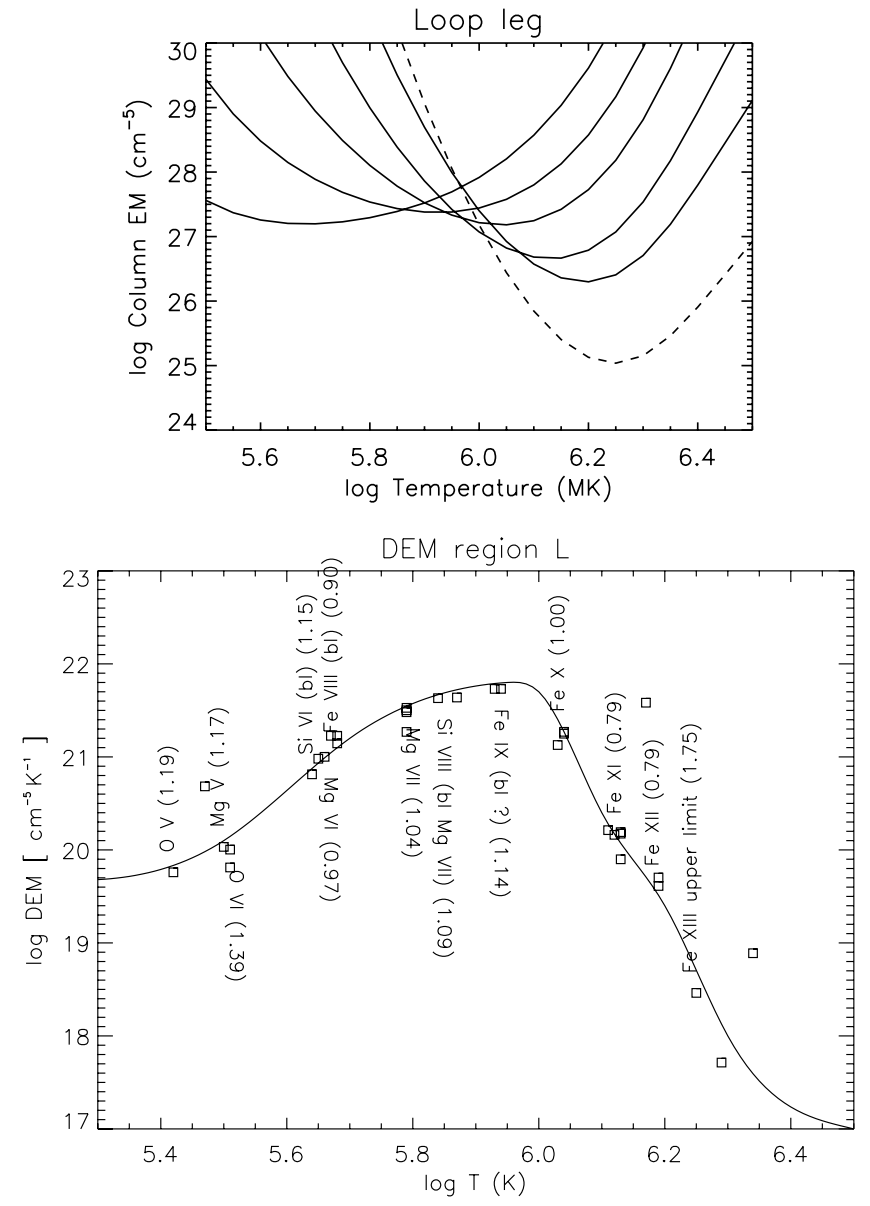

Fig. 15. Top: EM loci curves for the background-subtracted loop leg. Lines are from Fe viII, Fe Ix, Fe x, Fe xi and Fe xII. The dashed line is an upper limit for Fe xIII. Bottom: the corresponding DEM for the loop leg.

We have then simulated the AIA count rates as done previously, and found that in this case the contribution from lines formed below $1 \mathrm{MK}$ is even more dominant, as is expected from the shape of the DEM. Figures C.1, C.2 in the Appendix show the simulated AIA count rates based on the DEM modeling. Tables C.1, C.2 also in the Appendix show the main lines contributing to the AIA count rates in the 193 and $211 \AA$ channels, obtained as in the previous case.

Any analysis based on AIA observations of warm loops will be very uncertain, considering the large number of unidentified cool TR lines, and the uncertain atomic data for those lines that are known. The total AIA simulated count rates based on the DEM modeling are shown in Table 4. As in the previous case, relatively good agreement is found between the observed and simulated count rates for the 131, 193, and $335 \AA$ bands, but large discrepancies are present for the 94, 171, and $211 \AA$ bands.

\section{DEM inversion using the AIA data}

We now assess if it is possible to derive accurate emission measures from the AIA observations, using the cool footpoint region and the loop leg as examples. First of all, given that the Del Zanna (1999) DEM analysis method, despite being robust, is subjective in the choice of the nodes of the spline, we have also carried out a DEM analysis with the same Hinode/EIS data using the MCMC method (Kashyap \& Drake 1998). We wrote custom- 
Table 5. Observed and predicted (from DEM modeling) SDO/AIA count rates for region " $\mathrm{B}$ ".

\begin{tabular}{lccccc}
\hline \hline $\begin{array}{l}\text { Band } \\
(\AA)\end{array}$ & Obs. & $\begin{array}{c}\text { Pred. } \\
(6 \text { bands })\end{array}$ & \multicolumn{3}{c}{$\begin{array}{c}\text { Pred. } \\
\text { (4 bands) }\end{array}$} \\
\hline 94 & 22 & 15.9 & $(-28 \%)$ & - & $(-0.07 \%)$ \\
131 & 422 & 416 & $(-1.4 \%)$ & 422 & $(-0.39 \%)$ \\
171 & 7323 & 7540 & $(2.9 \%)$ & 7294 & $(0.25 \%)$ \\
193 & 4163 & 4660 & $(12 \%)$ & 4173 & \\
211 & 1363 & 1220 & $(-11 \%)$ & - & $(-0.06 \%)$ \\
335 & 93 & 105 & $(13 \%)$ & 92.9 & $(-0.05 \%)$ \\
\hline
\end{tabular}

Notes. The AIA count rates are in averaged DN/s per EIS pixel. AIA DEM modeling was performed for two separate cases. One of which used six AIA bands, while the other used four. The latter case excluded the $94 \AA$ and $211 \AA$ bands. Percentage differences are included in parentheses.

Table 6. Same as Table 5 for the background-subtracted loop leg.

\begin{tabular}{lccccc}
\hline \hline $\begin{array}{l}\text { Band } \\
(\AA)\end{array}$ & Obs. & $\begin{array}{c}\text { Pred. } \\
\text { (6 bands) }\end{array}$ & \multicolumn{3}{c}{$\begin{array}{c}\text { Pred. } \\
\text { (4 bands) }\end{array}$} \\
\hline 94 & 9 & 3.3 & $(-63 \%)$ & - & \\
131 & 102 & 97 & $(-5.0 \%)$ & 102 & $(0.02 \%)$ \\
171 & 1449 & 1546 & $(6.7 \%)$ & 1445 & $(-0.27 \%)$ \\
193 & 821 & 925 & $(13 \%)$ & 824 & $(0.41 \%)$ \\
211 & 266 & 228 & $(-14 \%)$ & - & $(-0.25 \%)$ \\
335 & 18.6 & 21.5 & $(16 \%)$ & 18.6 & $(-20)$ \\
\hline
\end{tabular}

written software to run the MCMC code. The MCMC routine is robust and has been used for a long time to study stellar coronae. The AIA response functions have been obtained using the same set of atomic data and parameters, as described in the Appendix.

The result for the cool footpoint region B using the MCMC method and EIS data, shown in Fig. 16 (top), is very similar to that given in Fig. 6. The fitting was carried out over the temperature range $\log T[\mathrm{~K}]=5.5-6.5$ and with a $\log T[\mathrm{~K}]=0.1$ step.

On the other hand, a very different DEM distribution is obtained when the MCMC inversion technique is applied to the AIA count rates. We first applied the inversion using all six EUV bands (Fig. 16, middle), then excluding the 94 and $211 \AA$ bands (Fig. 16, bottom), which we know are unreliable. In both cases, very different DEM distributions are obtained, despite the fact that all the AIA count rates are closely reproduced, as shown in detail in Table 5.

The results for the loop leg region $\mathrm{L}$ are somewhat similar. Any DEM inversion of an almost isothermal plasma is going to be particularly challenging. Fig. 16 (top) shows the result obtained with the MCMC method and a selection of EIS spectral lines. The method does provide a clear peak at the correct temperature, although it overestimates the DEM at lower $T$. It can be compared with Fig. 15.

On the other hand, as in the previous case, a completely different DEM distribution is obtained when the SDO/AIA data are used, using the six and four bands (Fig. 16 middle, bottom). It should be noted that despite the fact that the AIA DEMs are very different, as in the previous case, the AIA count rates are very closely reproduced, with the exception of the $94 \AA$ as shown in Table 6.
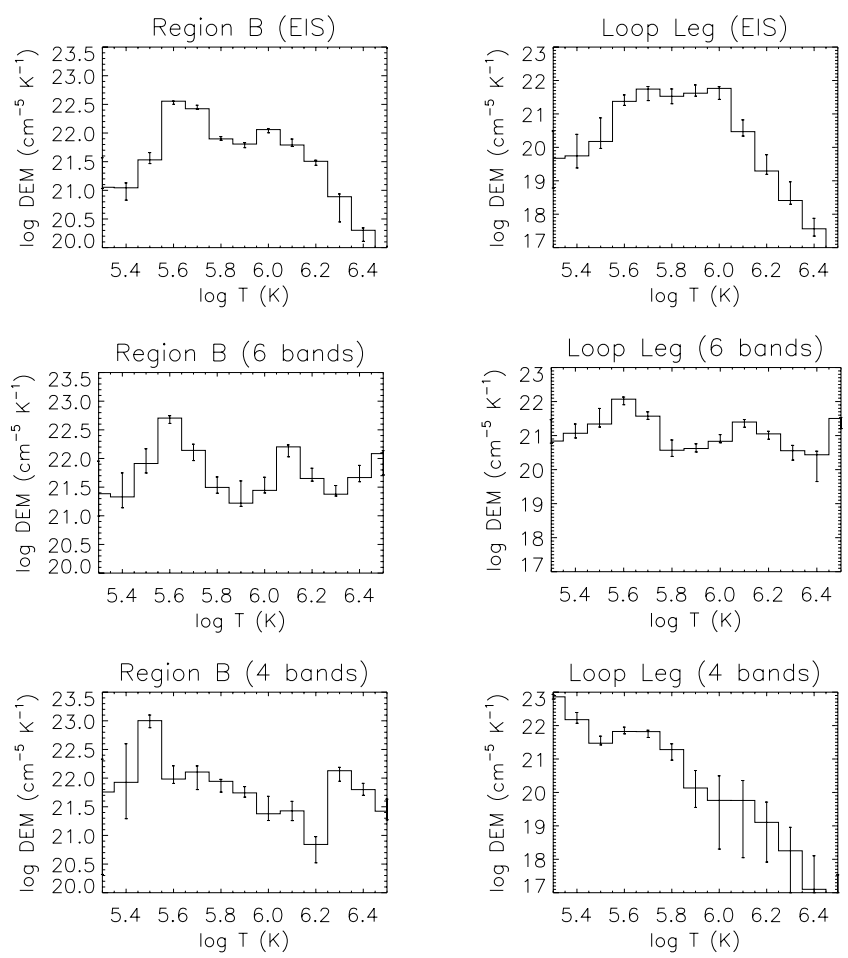

Fig. 16. Top: the DEM derived from Hinode/EIS with the MCMC method. Middle: the DEM obtained from the SDO/AIA data with all six bands. Bottom: the DEM obtained from the SDO/AIA data, excluding the 94 and $211 \AA$ bands. Left: the loop foot-point region "B". Right: the background-subtracted loop region " $L$ ".

\section{Conclusions}

We confirm the results predicted by O'Dwyer et al. (2010), in that all the AIA EUV bands are naturally multi-thermal. In particular, we have extended our previous analysis to study the contribution of cool emission to the AIA bands. We presented a cool loop footpoint and a warm loop leg. The DEM for the cool loop footpoint peaked at $\log T[\mathrm{~K}]=5.7$ and for the warm loop leg at around $\log T[\mathrm{~K}]=5.9$. We have carried out a direct (with overlapping wavelength ranges) and indirect (by deriving a DEM from EIS and forward modelling) simulation of AIA channels.

We find significant contributions from lines formed from log $T[\mathrm{~K}]=5.2$ (e.g. O v), 5.4 (e.g. Fe vII), 5.6 (e.g. Fe vIII), and 5.7 (e.g. Fe Ix) in the 94, 131, 193, 211, $335 \AA$ channels. The contribution of cool $(\log T[\mathrm{~K}]<5.7)$ plasma to the $171 \AA$ channel is also clearly significant. Our results suggest that the interpretation given by De Pontieu et al. (2011) of the AIA $171 \AA$ and $211 \AA$ observations near active region loop footpoints should be treated with extreme caution. Indeed we have shown that for both a loop footpoint and a loop leg region, the contribution of "cool" (log $T[\mathrm{~K}]<5.7)$ plasma is large. For the loop footpoint region, more than $50 \%$ of the AIA counts in the $211 \AA$ channel as observed by EIS are due to "cool" lines. For the loop leg case, 30\% (40\%) of the AIA counts in the $193 \AA$ ( $211 \AA$ ) channel, as observed by EIS, are due to "cool" lines.

It is therefore plausible that the few brightenings in the AIA 304, 171, and $211 \AA$ bands that we and De Pontieu et al. (2011) observe near the footpoint of active region loops are signatures of upflowing chromospheric material heated to transition-region temperatures, up to $0.5 \mathrm{MK}$, although the EIS 
observations presented here cannot rule out heating to even higher temperatures.

The relatively good agreement (to within a relative 20\%) between the EIS and AIA observations in the $193 \AA$ channel is encouraging, considering that it is based on older and independent laboratory calibration measurements performed years apart (see also O'Dwyer et al. 2011, for an earlier AIA/EIS comparison).

The atomic data for many ions, which are important for AIA, need improvement. We find that many unidentified (both cool and coronal) lines contribute to the AIA channels. For the footpoint region, more than $50 \%$ of the AIA counts in the 211 are due to unidentified lines. For both the loop footpoint and the loop leg case, $17 \%(50 \%)$ of the AIA counts in the $193 \AA$ (211 $\AA$ ) channel are due to unidentified lines for which we do not have atomic data. For the loop footpoint case, 32\% (20\%) of the AIA counts in the $193 \AA$ ( $211 \AA)$ channel are due to "cool" lines for which many atomic data/identifications are still uncertain. For the loop leg case, $25 \%(12 \%)$ of the AIA counts in the $193 \AA(211 \AA)$ channel are due to "cool" lines with uncertain atomic data.

We have already carried out a significant amount of atomic physics work in this regard and more work is in progress for Fe viII, Fe Ix, and Fex. It is clear from our analysis that the 94 and $211 \AA$ bands should not be used for quantitative diagnostic purposes. We have also highlighted significant problems in the 131, 171 A channels, which should be used with caution.

Different source regions in the solar atmosphere have very different spectral signatures. We stress that even the results shown here should not be generalised. We have found that the inversion of the AIA data to obtain a description of the thermal characteristics of warm loops is unreliable. The inability to recover the DEM is probably related to the fact that the AIA responses (cf. Fig. 1) are much wider in temperature, compared to single EIS iron lines (with the exception of the $171 \AA$ ). Another possibility is the fact that most AIA responses are doublepeaked. More tests will be required to ascertain the reasons for the large discrepancies between the derived DEMs.

Acknowledgements. G.D.Z. acknowledges support from STFC (UK) via the Advanced Fellowship programme. B.O.D. and H.E.M. also acknowledge support from STFC. B.O.D. was supported by funding from the Gates Cambridge Trust. The AIA data used here are courtesy of SDO (NASA) and the AIA consortium.
Hinode is a Japanese mission developed and launched by ISAS/JAXA, with NAOJ as domestic partner and NASA and STFC (UK) as international partners. It is operated by these agencies in co-operation with ESA and NSC (Norway). The EIS observations were requested at the 2010 EIS meeting and designed in collaboration with P. Young and H. Warren. CHIANTI is a collaborative project involving researchers at the Universities of Cambridge (UK), George Mason, and Michigan (USA).

\section{References}

Aschwanden, M. J., \& Boerner, P. 2011, ApJ, 732, 81

Asplund, M., Grevesse, N., Sauval, A. J., \& Scott, P. 2009, ARA\&A, 47, 481 Berghmans, D., \& Clette, F. 1999, Sol. Phys., 186, 207

Boerner, P., Edwards, C., Lemen, J., et al. 2011, Sol. Phys., 193

Culhane, J. L., Harra, L. K., James, A. M., et al. 2007, Sol. Phys., 60 De Pontieu, B., McIntosh, S. W., Carlsson, M., et al. 2011, Science, 331, 55

Del Zanna, G. 1999, Ph.D. Thesis, Univ. of Central Lancashire, UK

Del Zanna, G. 2003a, A\&A, 406, L5

Del Zanna, G. 2003b, A\&A, 406, L5

Del Zanna, G. 2009a, A\&A, 508, 501

Del Zanna, G. 2009b, A\&A, 508, 513

Del Zanna, G. 2010, A\&A, 514, A41

Del Zanna, G., \& Mason, H. E. 2003, A\&A, 406, 1089

Del Zanna, G., \& Ishikawa, Y. 2009, A\&A, 508, 1517

Del Zanna, G., Landini, M., \& Mason, H. E. 2002, A\&A, 385, 968

Del Zanna, G., Mason, H. E., \& Cirtain, J. 2006, in ESA SP, 617, 86

Del Zanna, G., Storey, P. J., \& Mason, H. E. 2010, A\&A, 514, A40

Dere, K. P., Landi, E., Young, P. R., et al. 2009, A\&A, 498, 915

Hara, H. 2008, in First Results from Hinode, ed. S. A. Matthews, J. M. Davis, \& L. K. Harra, ASP Conf. Ser., 397, 11

Kashyap, V., \& Drake, J. J. 1998, ApJ, 503, 450

Landi, E., Del Zanna, G., Young, P. R., Dere, K. P., \& Mason, H. E. 2011, ApJ, accepted

Lang, J., Kent, B. J., Paustian, W., et al. 2006, Appl. Opt., 45, 8689

Lemen, J., Title, A., Akin, D., et al. 2011, Sol. Phys., submitted

Liang, G. Y., Badnell, N. R., Crespo López-Urrutia, J. R., et al. 2010, ApJS, 190, 322

O’Dwyer, B., Del Zanna, G., Mason, H. E., Weber, M. A., \& Tripathi, D. 2010, A\&A, 521, A21

O’Dwyer, B., Del Zanna, G., Mason, H. E., Weber, M. A., \& Tripathi, D. 2011, Proc. Hinode 4 , in press

Schmelz, J. T., Kimble, J. A., Jenkins, B. S., et al. 2010, ApJ, 725, L34

Schmelz, J. T., Jenkins, B. S., Worley, B. T., et al. 2011, ApJ, 731, 49

Schrijver, C. J., Title, A. M., Berger, T. E., et al. 1999, Sol. Phys., 187, 261

Strong, K. 1978, Ph.D. Thesis, University College London, UK

Witthoeft, M. C., \& Badnell, N. R. 2008, A\&A, 481, 543

Young, P. R., \& Landi, E. 2009, ApJ, 707, 173

Young, P. R., Del Zanna, G., Mason, H. E., et al. 2007, PASJ, 59, 727

Pages 13 to 16 are available in the electronic edition of the journal at http://www . aanda.org 


\section{Appendix A: AIA response functions}

Figure A.1 shows the AIA response functions calculated with the present atomic data (full lines) as compared to those obtained from CHIANTI v.6 (dashed lines).

Since iron is the dominant element in all of the six EUV bands considered here, the iron abundance is the main unknown paramater. The "coronal" abundances adopted within the standard AIA responses are a compilation of older measurements, and have an iron abundance a factor 3.98 higher than the photospheric value of Asplund et al. (2009). Any emission measure obtained from AIA observations would therefore scale by this factor. It is however interesting to see if different elemental abundances have an effect on the shape of the AIA responses. Figure A.2 displays the AIA response functions calculated with "coronal" abundances (solid lines) and with the 'photospheric' abundances, scaled by a 3.98 factor (dashed lines). It is clear that the main peaks in the responses are the same, due to the fact that the peak emission contributing to the AIA bands comes from iron. However, significant differences in the secondary peaks, in particular for the 193 and $211 \AA$ bands, are present. These differences would be enhanced when cool emission is observed.

\section{; Here is a simple example on how to calculate the \\ ; AIA temperature response function for the \\ 193 A channel, using the SSW AIA and \\ CHIANTI programs.}

; First, we need to define an array of
; temperatures:

temp $=10 \cdot d^{\wedge}($ indgen $(81) * 0.05+4.0)$

; The we calculate an isothermal spectrum using

; the CHIANTI routine isothermal.

; The example below is with constant density

; (edensity=1.e9), including all the lines

(/all),

; the continuum (/cont), over a range of

$5--500$ A,

; with a 0.1 A bin, and your choice of elemental

; (abund_name=) and ion (ioneq_name=) abundance.

isothermal, 5, 500, 0.1, temp, lambda, spectrum, $\$$ list_wvl, list_ident, $\$$

edensity=1.e9,/photons,/cont, \$

abund_name=' asplund_etal_09. abund', \$ , /all

ioneq_name $=$ ! xuvtop+' /ioneq/chianti . ioneq'

; This is the conversion factor for an AIA pixel

; (number of steradians per AIA pixel size):

sterad_aia_pix $=8.4 \mathrm{~d}-12$

; Get the AIA effective areas from Solarsoft:

aia_resp = aia_get_response $(/ d n)$

; regrid the AIA effective areas onto the

; wavelength grid with e.g. interpol:

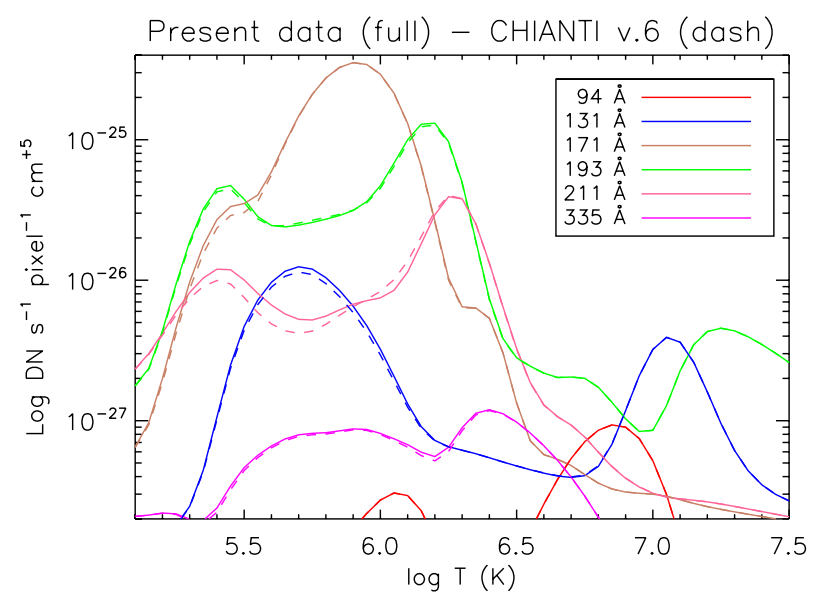

Fig. A.1. Top: the SDO AIA response functions calculated with the CHIANTI v.6 ion abundances, the "photospheric" (Asplund et al. 2009) abundances at constant pressure $\left(10^{15} \mathrm{~cm}^{-3} \mathrm{~K}\right)$, with the present atomic data (full lines) and CHIANTI v.6 (dashed lines).
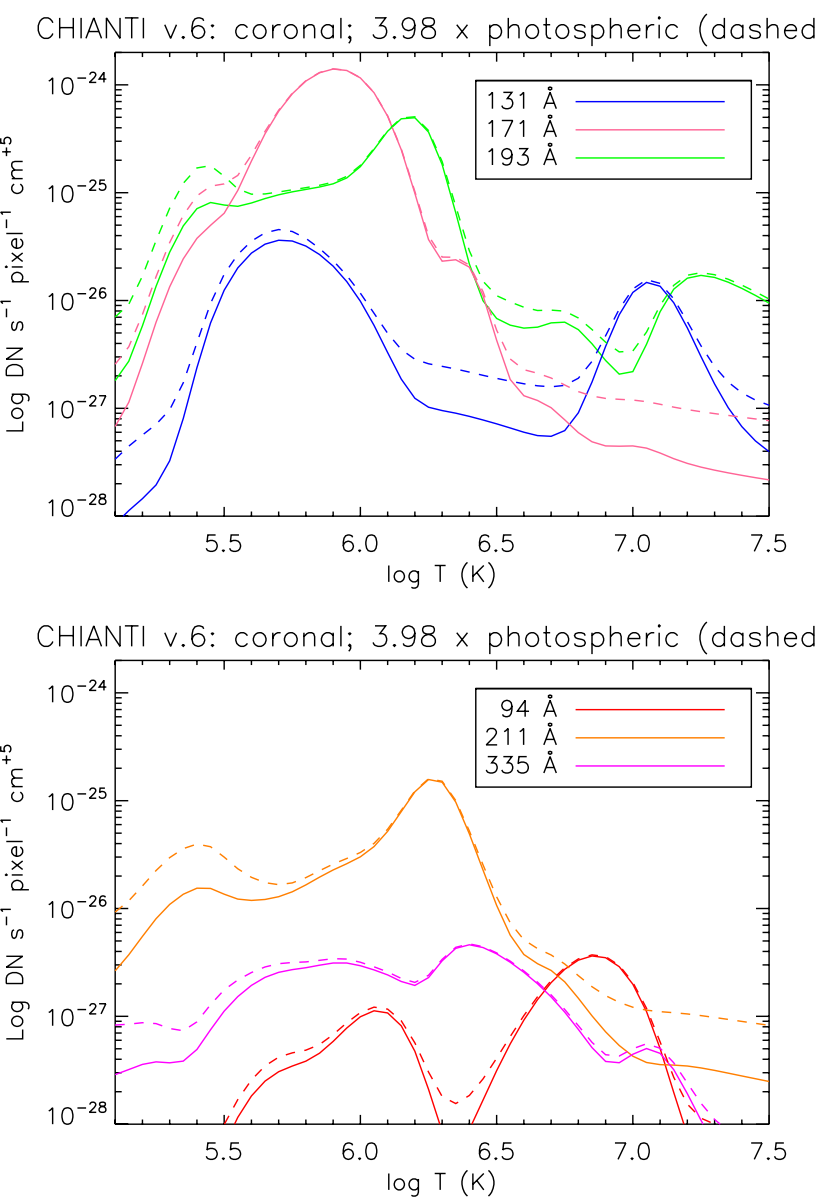

Fig. A.2. The SDO AIA response functions calculated with the CHIANTI v.6 ion abundances, constant pressure $\left(10^{15} \mathrm{~cm}^{-3} \mathrm{~K}\right)$, "coronal" abundances (solid lines) and with "photospheric" abundances, scaled by a 3.98 factor (dashed lines).

eff_193=interpol(aia_resp.a193.ea, \$
aia_resp.a193.wave, lambda)

; fold the isothermal spectra with the ; effective areas: 
$\mathrm{sp} \_$conv $=$spectrum \& sp_conv $[*, *]=0$.

for $i=0, n \_$elements (temp) -1 do $\$$

sp_conv [*, i] =sterad_aia_pix*spectrum[*,i] $*$ eff_193

; total over the wavelengths:

resp_193=total (sp_conv, 1)

; plot

plot_oo, temp,resp_193

\section{Appendix B: The EIS spatial resolution}

In principle, AIA images could be used to estimate the effective EIS spatial resolution. As clearly shown in this paper, all AIA images are multi-thermal, hence a direct comparison with the EIS monchromatic images is not possible. The only direct comparison that can be made is when considering the $193 \AA$ band. Indeed EIS does observe all the lines contributing to the AIA $193 \AA$ band.

For each EIS slit position, we first convolved each AIA $193 \AA$ image. We then averaged those AIA images taken during each EIS exposure, rebinned them onto the "EIS pixel" size, and obtained a slice of the corresponding averaged AIA image. We then built a time-averaged "rebinned" image for direct comparison with the EIS monochromatic images. Figure B.1 shows three AIA $193 \AA$ images. The first (top right) is obtained without convolution, while the other two (bottom row) are obtained by convolving the AIA images with a Gaussian PSF of 2, and 4 " full-width-half-maximum (FWHM). We also took the EIS calibrated spectra, and for each point multiplied them with the AIA $193 \AA$ effective area, and summed over wavelength, to obtain effective AIA DN/s per EIS pixel. The resulting image is shown in Fig. B.1 (top left). This image is very close, in morphology and count rates, to the AIA one convolved with a PSF between 2 and $4^{\prime \prime}$, if one considers the presence of the jitter.

What is remarkable is the agreement between the count rates predicted from the EIS spectra and those actually measured by AIA. This is shown in Fig. B.2, where a cut across the images is shown. It is interesting to note that the exact value of the EIS PSF is not relevant for the discussion in this paper, indeed the count rates obtained with a PSF of 2 or 4 " are very similar in most locations. A more detailed analysis of the EIS PSF is deferred to a future paper, once the AIA PSF is well-known.

\section{Appendix C: AIA simulated data for the loop leg region $L$}

Simulated AIA count rates have been obtained for the loop leg region $\mathrm{L}$ following the same procedure outlined for the loop base region "B". The results, shown in Figs. C.1, C.2, are similar, although the plasma is somewhat warmer.
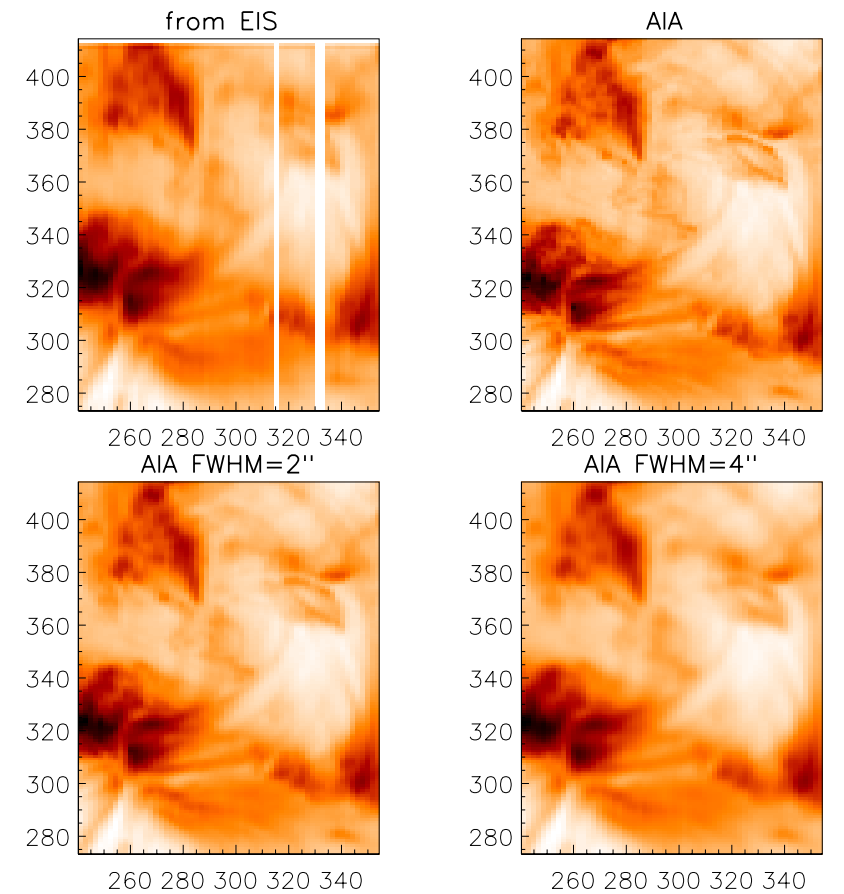

Fig. B.1. Top left: an image in the AIA $193 \AA$ band, as predicted from the Hinode EIS spectra. The other images are obtained from the AIA $193 \AA$ data, rebinned onto the EIS spatio-temporal scale. The top right is without convolution, while the other two are convolved with a PSF of FWHM of 2 and $4^{\prime \prime}$

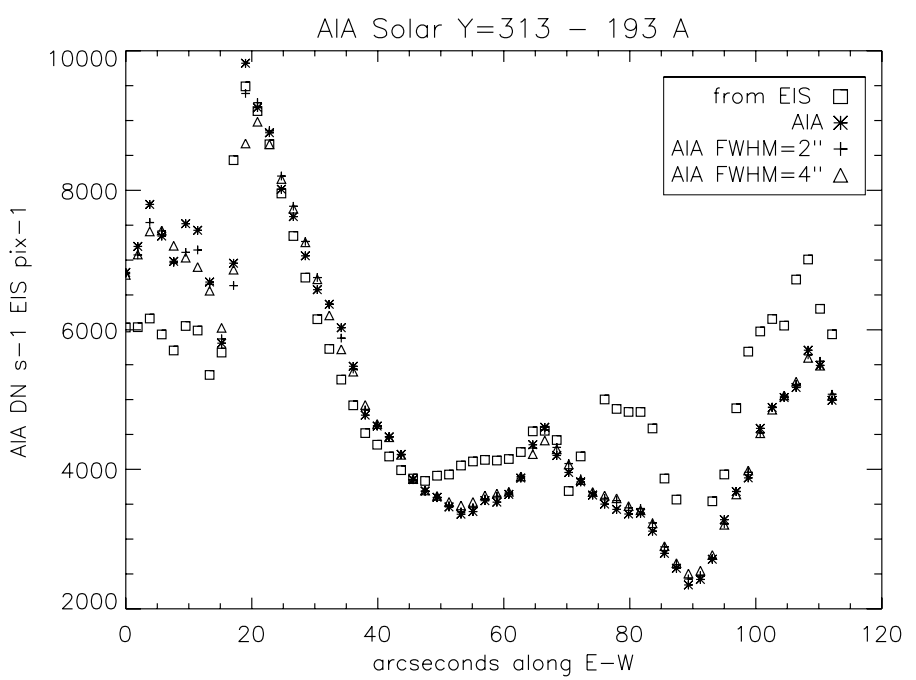

Fig. B.2. AIA $193 \AA$ count rates along the E-W direction, at solar $Y=313$ (see Fig. B.1). 
G. Del Zanna et al.: SDO AIA and Hinode EIS observations of "warm" loops
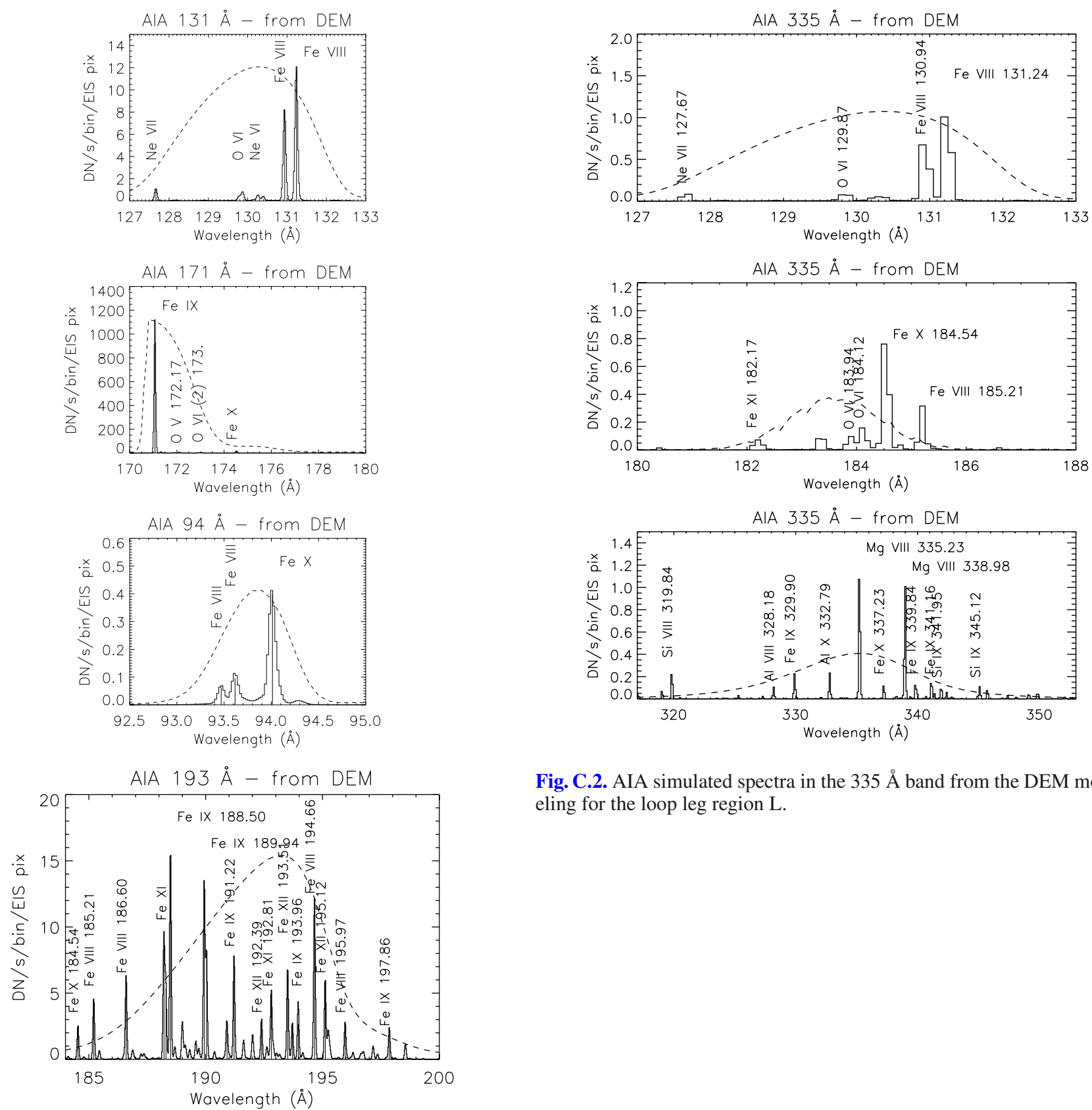

Fig. C.2. AIA simulated spectra in the $335 \AA$ band from the DEM modeling for the loop leg region L.

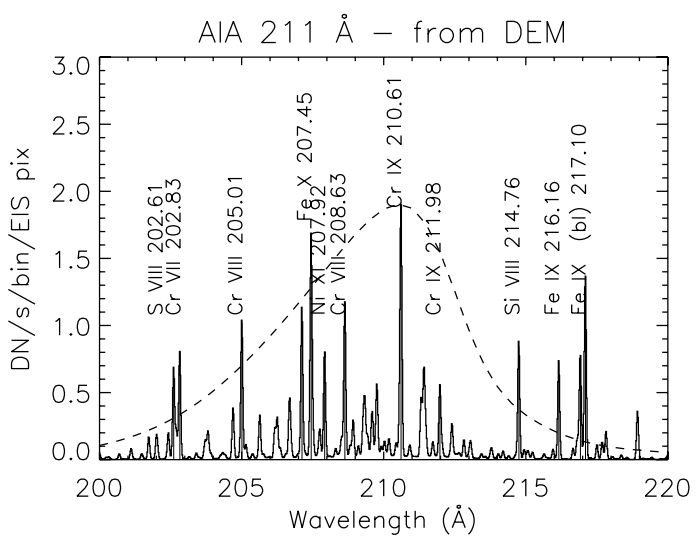

Fig. C.1. AIA simulated spectra from the DEM modeling for the loop leg region $\mathrm{L}$. 
Table C.1. List of the main Hinode EIS spectral lines contributing to the SDO AIA $193 \AA$ channel in the loop leg region L, as in Table 2.

\begin{tabular}{lcccl}
\hline \hline$\lambda_{\mathrm{o}}$ & DN (EIS) & R (EIS) & CR (AIA) & ID \\
\hline 184.55 & 2309 & 100 & 22 & Fe X \\
185.24 & 2805 & 102 & 32 & Fe VIII \\
186.63 & 3001 & 80 & 54 & Fe VIII \\
186.88 & 1277 & 32 & 25 & Fe XII (bl) \\
187.27 & 285 & 7 & 6 & Fe VIII \\
187.97 & 545 & 11 & 14 & u \\
188.23 & 5509 & 108 & 149 & Fe XI \\
188.31 & 3145 & 61 & 87 & Fe XI \\
188.38 & 391 & 7 & 11 & u (TR) \\
188.51 & 3158 & 59 & 90 & Fe IX \\
188.65 & 344 & 6 & 10 & u \\
188.83 & 325 & 6 & 10 & u (XI) \\
189.01 & 315 & 5 & 10 & Fe XI \\
189.14 & 353 & 6 & 11 & Fe XI \\
189.60 & 273 & 4 & 9 & u \\
189.74 & 334 & 5 & 11 & Fe XI \\
189.96 & 2382 & 36 & 83 & Fe IX \\
190.05 & 2979 & 45 & 105 & Fe X (Fe XI $?)$ \\
190.41 & 294 & 4 & 11 & Fe XI \\
190.92 & 262 & 4 & 10 & u (X bl TR) \\
191.24 & 1319 & 17 & 51 & Fe IX (bl) \\
191.62 & 402 & 5 & 16 & u (tr Mn IX ?) \\
191.73 & 247 & 3 & 10 & u \\
192.03 & 849 & 10 & 34 & Fe XI (bl) \\
192.11 & 751 & 9 & 30 & Fe VIII (?) \\
192.20 & 546 & 6 & 22 & u (X) \\
192.31 & 428 & 5 & 17 & u \\
192.40 & 2703 & 31 & 108 & Fe XII \\
192.65 & 1274 & 15 & 51 & u (X) \\
192.82 & 3018 & 34 & 120 & Fe XI (bl) \\
192.93 & 728 & 8 & 29 & O V \\
193.29 & 540 & 6 & 21 & u \\
193.52 & 6556 & 70 & 250 & Fe XII \\
193.73 & 1465 & 15 & 54 & Fe X \\
193.98 & 378 & 4 & 13 & Fe VIII \\
194.33 & 457 & 5 & 15 & u \\
194.68 & 3280 & 33 & 93 & Fe VIII \\
194.82 & 1150 & 12 & 30 & u (TR) \\
195.13 & 12039 & 120 & 267 & Fe XII \\
195.41 & 1421 & 14 & 26 & u (TR) \\
195.99 & 2300 & 23 & 26 & Fe VIII (bl) \\
196.67 & 1441 & 15 & 10 & Fe XII \\
197.88 & 3196 & 36 & 14 & Fe IX \\
& & & & \\
& Totals & & 110 & u (TR) \\
& & & 563 & TR \\
& & & 290 & u (Coronal) \\
\hline & & & 1314 & Coronal \\
\hline 19 & & &
\end{tabular}

Table C.2. List of the main Hinode EIS spectral lines contributing to the SDO AIA $211 \AA$ channel in the loop leg region L, as in Table 2.

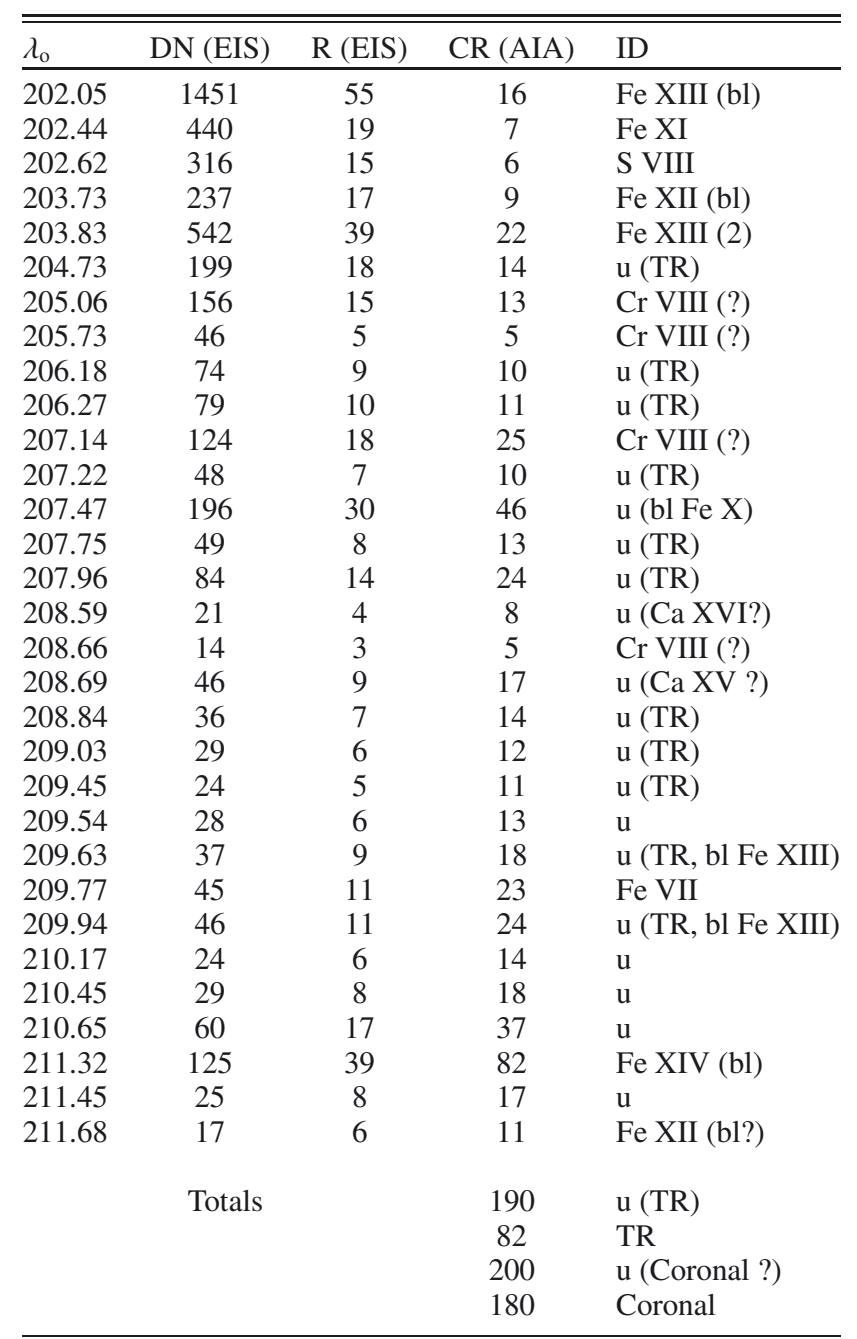

